Preprint typeset in JHEP style - HYPER VERSION

KCL-MTH-02-25

DAMTP-2002-139

hep-th/0211122

\title{
The $D$-instanton and other supersymmetric $D$-branes in IIB plane-wave string theory
}

\author{
Matthias R. Gaberdiel* \\ School of Natural Sciences, Institute for Advanced Study \\ Princeton, NJ 08540, USA \\ E-mail: mrg@sns.ias.edu, mrg@mth.kcl.ac.uk \\ Michael B. Green \\ Department of Applied Mathematics and Theoretical Physics \\ Wilberforce Road, Cambridge CB3 0WA, UK \\ E-mail: M.B.Green@damtp.cam.ac.uk
}

\begin{abstract}
A class of $D$-branes for the type IIB plane-wave background is considered that preserve half the dynamical supersymmetries of the light-cone gauge. The $D$-branes of this type are the euclidean (or instantonic) $(0,0),(0,4)$ and $(4,0)$ branes (where $(r, s)$ denotes a brane oriented with $r$ axes in the first four directions transverse to the,+- light-cone and $s$ axes in the second four directions). Corresponding lorentzian $D$-branes are $(+,-; 0,0)$, $(+,-; 0,4)$ and $(+,-; 4,0)$. These are constructed in two ways. The first uses a boundary state formalism which implements appropriate fermionic gluing conditions and the second is based on a direct quantisation of the open strings ending on the branes. In distinction to the $D$-branes considered earlier these have massless world-volume fermions but do not possess kinematical supersymmetries. Cylinder diagrams describing the overlap between a pair of boundary states displaced by some distance are evaluated. The open-string description of this system involves mode frequencies that are, in general, given by irrational solutions to transcendental equations. The closed-string and open-string descriptions are shown to be equivalent by a nontrivial implementation of the $S$ modular transformation. A classical description of the $D$-instanton (the $(0,0)$ case) in light-cone gauge is also given.
\end{abstract}

KEYWORDS: Superstring.

${ }^{*}$ On leave of absence from: Department of Mathematics, King's College London, Strand, London WC2R 2LS, UK. 


\section{Contents}

1. Introduction and review 2

2. The $(0,0)$ (D-instanton) boundary state and its overlaps 0

2.1 Cylinder diagrams involving the $D$-instanton 10

3. The open string point of view 12

3.1 Boundary conditions and mode expansions 12

$3.1 .1 \quad(0,0)-(0,0)$

$3.1 .2(0,0)-\overline{(0,0)}$

$3.1 .3(0,0)-(r, r+2)$

3.2 Consistency of one-loop open-string amplitudes 16

4. $(4,0)$ and $(0,4)$ with flux 18

4.1 The open string description 19

4.2 Supersymmetry 20

5. Relation to $(2,2)$ world-sheet supersymmetry 21

6. The classical $D$-instanton 24

6.1 Review of the flat space description 24

6.2 The classical solution for the plane-wave background 26

6.3 Supergravity approximation of the interaction energy 27

7. Discussion 29

A. Notation and definitions 30

B. Definition of $f_{i}, g_{i}, \hat{g}_{i}, g_{i, \pm}, \hat{g}_{i, \pm}$

B.1 The factorized $g$-functions 35

G. Supersymmetry of the open string 36

C.1 Anti-commutation relations of the modes 36

C.2 Dynamical supercharge 37

D. Modular properties of the cylinder diagrams 39

D.1 Closed-string perspective 39

D.2 Open-string perspective 


\section{Introduction and review}

The study of type IIB superstring theory in $p p$-wave backgrounds is a fertile area for investigating the properties of string theory with nontrivial Ramond-Ramond $(R R)$ condensates. In the simplest case, the plane-wave background with a constant flux of the Ramond-Ramond $(R R)$ five-form field strength, there are 32 supersymmetries, which is the maximal number. Perturbative superstring theory in this background was formulated in [1, 2]. The metric has a $S O(4) \times S O(4)$ isometry group which distinguishes the directions $x^{I}$ with $I=i=1,2,3,4$ from the directions with $I=i^{\prime}+4=5,6,7,8$. In this notation the light-cone directions are $x^{ \pm}=\left(x^{0} \pm x^{9}\right) / \sqrt{2}$, where $x^{0}$ is time-like. One of the main interests in studying such theories is the connection, via the Penrose limit [3], with $\mathcal{N}=4$ supersymmetric Yang-Mills theory 细. A variety of generalizations of the maximally supersymmetric plane-wave with less supersymmetry have been constructed. Particularly interesting is the class of theories that are based on $(2,2)$ world-sheet supersymmetry [5]. These backgrounds have been shown to be exact solutions of superstring theory to all orders in $\alpha^{\prime}$ [6]. In these cases the generic background, which is expressed in terms of a superpotential, has less space-time supersymmetry and the string theory is governed by an integrable two-dimensional system.

This paper will continue the study of $D$-branes which preserve half of the dynamical supersymmetry of the maximally supersymmetric plane-wave background. For much of the time we will be discussing instantonic $D$-branes, which are defined by euclidean embeddings of $(p+1)$-dimensional world-volumes. These are the cases in which the light-cone directions $x^{ \pm}$are orthogonal to the brane. We will adopt a notation (similar to that in [7], which provides a useful overview of various $D$-branes that arise in this background) in which these instantonic branes are denoted $(r, s)$-branes $(r+s=p+1)$, where $r$ and $s$ are the numbers of directions associated with the two $S O(4)$ factors in the transverse space. Our considerations apply equally well to $D p$-branes with lorentzian signature which will be denoted $(+,-; r, s)$-branes $(r+s=p-1)$. Recently, it has been found that there are also various 'oblique' branes which are constrained to be oriented in directions that couple the two $S O(4)$ 's [8]. Such branes, which cannot be classified as $(r, s)$-branes, possess less supersymmetry than those considered here. They arise naturally in the backgrounds of [5].

In section 2 we will present the construction of the branes in a light-cone formalism that extends the discussions of [9]. From the closed-string perspective this generalizes the flat-space construction in [10], and starts with the ansatz that the boundary state describing the brane is annihilated by half the 'dynamical' light-cone supercharges ${ }^{1}$,

$$
\left.\left.\left(Q_{\dot{a}}+i \eta M_{\dot{a} \dot{b}} \tilde{Q}_{\dot{b}}\right) \|(r, s), \eta\right\rangle\right\rangle=0
$$

where the value of $\eta= \pm 1$ distinguishes a brane from an anti-brane. We will also assume that the bosonic coordinates satisfy the standard boundary conditions in Dirichlet directions (but not necessarily in Neumann directions since these may be affected by the background $R R$ flux). As we will see these conditions also imply that the boundary state

\footnotetext{
${ }^{1}$ Conventions and notation are explained in appendix D.
} 
preserves half the 'kinematical' light-cone supercharges,

$$
\left.\left.\left(Q_{a}+i \eta M_{a b} \tilde{Q}_{b}\right) \|(r, s), \eta\right\rangle\right)=0 .
$$

This means that the fermionic modes satisfy the gluing conditions

$$
\left.\left(S_{0}^{a}+i \eta M_{a b} \tilde{S}_{0}^{b}\right) \|(r, s), \eta\right\rangle=0, \quad k \in \mathbb{Z} .
$$

Importantly, we will not assume that the same condition holds for the non-zero modes of the supercharge density, which was the case in [11] and [9].

As in the flat-space case the matrix $M$ is given by

$$
M_{a b}=\left(\prod_{I \in \mathcal{N}} \gamma^{I}\right)_{a b}, \quad M_{\dot{a} \dot{b}}=\left(\prod_{I \in \mathcal{N}} \gamma^{I}\right)_{\dot{a} \dot{b}},
$$

in the two inequivalent $S O(8)$ spinor representations. Here $\mathcal{N}$ denotes the set of directions for which the brane satisfies a 'Neumann' boundary condition. We will see that in the $p p$ wave background there are two classes of maximally supersymmetric $D p$-branes, depending on the choice of $M$ :

- Class I. The first class is the one that was studied in [11, 12, 9] and arises when the matrix $M_{a b}$ satisfies

$$
\Pi M \Pi M=-1,
$$

where $\Pi=\gamma^{1} \gamma^{2} \gamma^{3} \gamma^{4}$. This condition was considered in the cases of instantonic $D$-branes in [11] and lorentzian $D$-branes in [12]. The branes of this kind are of the form $(r, r+2)$ and $(r+2, r)$. They preserve half of the dynamical supersymmetries as well as half of the kinematical supersymmetries. There are two types of $M$ that satisfy (1.5),

$$
\Pi M=-M \Pi, \quad M^{T}=M,
$$

which includes the cases $(3,1),(1,3)$, or

$$
\text { (ii) } \quad \Pi M=M \Pi, \quad M^{T}=-M,
$$

which includes the cases $(2,0),(0,2),(4,2),(2,4)$. In all of these cases the open-string sector preserves eight components (i.e. half) of both the dynamical and kinematical supersymmetries. A characteristic feature of this class is that the kinematical conditions (1.2) are not preserved as a function of $x^{+}$since the commutator with the light-cone hamiltonian has the form

$$
\left[H, Q_{a}+i \eta M_{a b} \tilde{Q}_{b}\right]=\frac{m \eta}{2 p^{+}}\left(\Pi M^{t}\right)_{a b}\left(Q_{b}-i \eta M_{b c} \tilde{Q}_{c}\right) .
$$

In this case the open-string theory has a mass term in its hamiltonian of the form $S_{0} M \Pi S_{0}$ [12], and the ground state is an unmatched boson.

- Class II. The second class arises when the matrix $M_{a b}$ satisfies

$$
\Pi M \Pi M=1
$$


a possibility that was not considered in [11, 12, 9] but arose in the supergravity analyses of [7, 13]. Those branes in this class which preserve half of the dynamical supersymmetries possess no open-string kinematical supersymmetries. There are two types of $M$ satisfying (1.9),

$$
\text { (i) } \quad \Pi M=M \Pi, \quad M^{T}=M,
$$

which includes the cases $(0,0),(2,2),(4,0),(0,4),(4,4)$ or

$$
\Pi M=-M \Pi, \quad M^{T}=-M .
$$

which includes the cases $(1,1),(3,3)$. In these cases

$$
\left[H, Q_{a}+i \eta M_{a b} \tilde{Q}_{b}\right]=-\frac{m \eta}{2 p^{+}}\left(\Pi M^{t}\right)_{a b}\left(Q_{b}+i \eta M_{b c} \tilde{Q}_{c}\right)
$$

and thus the kinematical conditions (1.2) are preserved as a function of $x^{+}$. In this case $S_{0} M \Pi S_{0} \equiv 0$ and the open-string mass term vanishes. The ground states then form a degenerate supermultiplet.

In section 2 we will obtain the boundary states for the (0,0)-brane (or $D$-instanton), which is a class II brane. We will also evaluate the overlap of the $(0,0)$ with itself, with the $\overline{(0,0)}$ (the anti $D$-instanton), and with $(r, r+2)$-branes. In this manner we determine the cylinder diagrams that describe the interaction energies (more accurately, the interaction actions') of these instantonic objects.

The relevant cylinders have parameter length $X^{+}$, which is the difference between the $x^{+}$positions of the two instantonic $D$-branes, and circumference $2 \pi p^{+}$. The relevant modular parameter of the cylinder is therefore $X^{+} / 2 \pi p^{+}$. The light-cone gauge in the closed-string channel is chosen such the $x^{+}$is proportional to the world-sheet time coordinate $\tau$ and thus $\tau$ parametrises the direction along the finite length of the cylinder, while the world-sheet space coordinate $\sigma$ is the periodic variable along the circular direction. In order to understand the relation to the open-string description it is necessary to perform a Wick rotation, replacing $\tau$ by $i \tau$ as well as $x^{+}$by $i x^{+}$, so that the world-sheet theory, as well as the space-time theory, become euclidean. (While it is not clear how to perform the Penrose limit for a euclidean theory, it is certainly possibly to Wick rotate the resulting plane wave background.) The two world-sheet coordinates $\sigma$ and $\tau$ then appear on an equal footing, and the cylinder diagram can be written as a function of the modular parameter (see [9] for more details)

$$
t=\frac{X^{+}}{2 \pi p^{+}}
$$

where $X^{+}$is the $x^{+}$separation between the two instantonic $D$-branes after the Wick rotation. $^{2}$ More specifically, for the class I branes discussed in [9], the cylinder diagrams can be expressed in terms of ratios of powers of functions $f_{i}^{(m)}(t)(i=1,2,3,4)$ whose definition is given in appendix B.

\footnotetext{
${ }^{2}$ That is,,$X^{+}=i X_{0}^{+}$, where $X_{0}^{+}$is the original distance between the $D$-instantons that is analytically continued to purely imaginary values in performing the Wick rotation.
} 
In order to identify the cylinder diagram with an open string one-loop diagram, the world-sheet parameters of the open string, $\tilde{\tau}$ and $\tilde{\sigma}$, are then identified with those of the closed string so that $\tilde{\tau}=\sigma$ and $\tilde{\sigma}=\tau$. This corresponds to choosing the light-cone gauge for the open string where $x^{+}$is proportional to $\tilde{\sigma}$. In the euclidean description where $\tilde{\tau}$ and $\tilde{\sigma}$ appear on the same footing, this is a consistent gauge choice.

In this open-string light-cone gauge the total length of the string in the $\tilde{\sigma}$ direction is $X^{+}$, while the proper time around the loop is $2 \pi p^{+}$. This means that $X^{+}$and $2 \pi p^{+}$are interchanged relative to the usual formulae that would hold for an open string ending on a time-like (lorentzian signature) brane. In particular, while the 'mass parameter' of the usual open string is $m=2 \pi \mu p^{+}$, the 'mass parameter' in the light-cone gauge appropriate to the instantonic branes is $\widehat{m}=\mu X^{+}$. Furthermore, the modular parameter in the open string description is

$$
\tilde{t}=\frac{1}{t}=\frac{2 \pi p^{+}}{X^{+}}
$$

The requirement that the cylinder diagram can equally well be described in terms of the open and closed string point of view implies that the amplitudes must transform appropriately under the $S$ modular transformation $t \mapsto \tilde{t}=1 / t$. For the case of the class I branes this turned out to be the case [9] because the functions $f_{i}^{(m)}$ transform as

$$
f_{1}^{(m)}(t)=f_{1}^{(\widehat{m})}(\tilde{t}), \quad f_{2}^{(m)}(t)=f_{4}^{(\widehat{m})}(\tilde{t}), \quad f_{3}^{(m)}(t)=f_{3}^{(\widehat{m})}(\tilde{t}),
$$

where $\widehat{m}=m t=\mu X^{+}$is the mass parameter in the open string description, as discussed above. In the limit $m \rightarrow 0$ these functions become the standard $f_{i}$ functions (except for a subtlety involving the zero modes for $f_{1}$ ) that are used to describe the cylinder diagrams of the flat space theory.

As usual, the discussion of section 2 does not fix the overall normalisation of the boundary states. This normalisation is left to be determined by relating the closed string calculation to the canonically normalised open string calculation as discussed above. The construction of the open string in section 3 is somewhat subtle compared to previous cases because the open string fermions have states with frequencies $\widehat{\omega}_{n}=\sqrt{n^{2}+\widehat{m}^{2}}$, where $n$ is generically an irrational number that is a solution of either

$$
\frac{n+i \widehat{m}}{n-i \widehat{m}}+e^{2 \pi i n}=0
$$

or

$$
\frac{n-i \widehat{m}}{n+i \widehat{m}}+e^{2 \pi i n}=0
$$

and $\widehat{m}=\mu X^{+}$is the mass-parameter in the open string light cone gauge. The space of solutions to the first of these equations will be called $\mathcal{P}_{+}$while the second equation defines the space $\mathcal{P}_{-}$. Although these equations also include the value $n=0$, this is not an allowed value for the open-string fermionic oscillators (as will be explained later).

In section 3.2 and appendix $D$ we will show that the open-string expression coincides with that derived from the boundary state approach, i.e. that the cylinder diagram transforms consistently under the $S$ modular transformation condition as in [9]. The fermionic 
contributions to the cylinder diagrams require further functions $g_{2}^{(m)}$ and $\widehat{g}_{4}^{(\widehat{m})}$. In appendix $\mathrm{B}$ it is explained that these functions transform into each other under the $S$ modular transformation,

$$
g_{2}^{(m)}(t)=\widehat{g}_{4}^{(\widehat{m})}(\tilde{t})
$$

The proof is considerably more subtle than that for the $f_{i}^{(m)}$ functions because the frequencies of the open string excitations in this case take the irrational values discussed above.

A similar analysis can also be performed for the lorentzian branes, as discussed in [9]. In this case the open-string light-cone gauge is the usual one in which $x^{+}=2 \pi \tilde{\tau} p^{+}$and, the parameter length of the string is $2 \pi p^{+}$. Following a Wick rotation, the closed-string description is one in which the length of the string in the $\sigma$-direction is now $X^{+}$, while the proper time interval is $2 \pi p^{+}$. Thus the 'mass parameter' in the open string is the usual $m$, while now the 'mass parameter' in the closed string is $\widehat{m}$. At any rate, the actual calculations are virtually identical to the corresponding calculations in the euclidean case, and we shall therefore not discuss them in detail.

In section 0 we will consider the cases of the $(0,4)$ and $(4,0)$ branes, starting from the conditions on the boundary states. In this case there is no consistent set of gluing conditions unless the bosons satisfy modified Neumann conditions in directions tangent to the brane. This is, of course, expected since these branes couple to the anti self-dual fourform background potential associated with the constant $R R$ five-form field strength. The $(0,4)$ and $(4,0)$ branes do not have supersymmetric anti-branes - the obvious candidates turn out to be branes for the theory in which the $R R$ four-form potential is self-dual instead of anti self-dual. The overlap between a $(4,0)$ and a separated $(2,0)$ is obtained and used to derive the appropriate boundary conditions on the open strings. The modified Neumann conditions translate into those expected on the basis of the Born-Infeld action and given in [7] and [8] (where they were obtained by requiring $(2,2)$ world-sheet supersymmetry).

The $(0,0),(4,0)$ and $(0,4)$ instantonic branes mentioned above seem to be the only class II branes that lead to a conserved dynamical supercharge in the open string. The same statements hold for the $(+,-; 0,0),(+,-; 4,0)$ and $(+,-; 0,4)$ lorentzian branes. Somewhat tantalizingly, there is another construction of a $(0,4)$ and $(4,0)$ boundary state, as well as of a $(4,4)$ boundary state, that satisfies (1.1) and (1.2) in the closed string sector. However, the corresponding open strings do not possess a conserved dynamical supercharge, and there are various indications that these boundary states do not actually satisfy the open-closed duality relation. It would be interesting to understand the meaning (if any) of these additional coherent states. Finally, from our analysis it seems that (1.1) and (1.2) do not have solutions in any of the other potential class II cases $((1,1),(2,2)$, and $(3,3))$, which agrees with the supergravity analysis in [13].

This conclusion is supported by the recent paper [8] which analyzed $D$-branes in the generalized $p p$-wave backgrounds of [5] that can be expressed in terms of $(2,2)$ world-sheet supersymmetry. The branes considered there are ones which preserve some fraction of this reduced supersymmetry. In section 5 we will argue that all of the potential class II examples are of this type, and that their presence or absence coincides with the results of 
[8]. The additional 'oblique' branes of that paper preserve less than eight components of the dynamical supersymmetry and should be seen by a small generalization of our boundary state gluing conditions although we have not done that.

As in the flat ten-dimensional case, the $(0,0)$-brane couples to the dilaton and the $R R$ pseudoscalar. In section 6 we will review the supergravity arguments that imply the presence of the $D$-instanton in flat space. We will also give an interpretation of the flatspace solution in the 'euclidean' light-cone gauge, i.e. after a Wick rotation in $x^{+}$. Making use of the conformal flatness of the plane-wave background together with the vanishing of the scalar curvature it is straightforward to see how the $(0,0)$-brane arises as a solution in the plane wave case. However, it is less obvious how to interpret the effect of this solution on the plane-wave dynamics. Finally, in section 7 we will review these results and make additional comments.

\section{The $(0,0)$ ( $D$-instanton) boundary state and its overlaps}

The class I boundary states were constructed in [11] in a manner that mimics the flat-space light-cone gauge construction [10] and preserves a complex combination of the closed-string supercharges. In this approach the light-cone $x^{ \pm}$directions are taken to be transverse to the brane world-volume which means that the description is appropriate for euclidean branes with $(p+1)$-dimensional world-volumes. The instantonic branes considered in [11] and [9] are of the form $(r, r+2)$ and $(r+2, r)$. A similar set of lorentzian signature $D p$-branes of the form $(+,-; r, r+2)$ and $(+,-; r+2, r)$, was obtained in 12 by analysis of the open-string sector. These cases are those of class I in the notation of section 1. In other words, they are ones for which the matrix $M$ satisfies $\Pi M \Pi M=-1$. In this section we will consider the boundary state approach for branes in class II where $M$ satisfies $\Pi M \Pi M=+1$. In particular, we will obtain a boundary state corresponding for the $(0,0)$ case (the $D$-instanton). This will be generalized in section 4 to the $(4,0)$ and $(0,4)$ cases (euclidean $D 3$-branes) which are special because they couple to the constant background self-dual $R R$ flux. These examples fit in well with the analysis of [8] which is based on $(2,2)$ world-sheet supersymmetry, whereas the class I branes are not invariant under this sub-symmetry (as will be discussed further in section 5).

We are interested in constructing a boundary state for a $(r, s)$-brane located at a transverse position $x_{0}^{I}=y_{t}^{I}$. The Dirichlet gluing conditions for the bosons take the form

$$
\begin{aligned}
\left.\left.\left(\alpha_{k}^{I}-\tilde{\alpha}_{-k}^{I}\right) \|(r, s), \mathbf{y}_{t}\right\rangle\right\rangle & =0, \quad k \in \mathbb{Z}, \\
\left.\left.\left(\bar{a}_{0}^{I}-a_{0}^{I}+i \sqrt{2 m} y_{t}^{I}\right) \|(r, s), \mathbf{y}_{t}\right\rangle\right\rangle & \left.\left.\equiv-i \sqrt{2 m}\left(x_{0}^{I}-y_{t}^{I}\right) \|(r, s), \mathbf{y}_{t}\right\rangle\right\rangle=0,
\end{aligned}
$$

where $I$ is a Dirichlet direction. Initially, the gluing conditions for the Neumann directions are assumed to be

$$
\begin{aligned}
\left.\left(\alpha_{k}^{J}+\tilde{\alpha}_{-k}^{J}\right) \|(r, s), \mathbf{y}_{t}\right\rangle & =0, \quad k \in \mathbb{Z} \\
\left.\left.\left(\bar{a}_{0}^{J}+a_{0}^{J}\right) \|(r, s), \mathbf{y}_{t}\right\rangle\right) & =0 .
\end{aligned}
$$


Furthermore, the boundary state must be annihilated by a certain linear combination of the dynamical supercharges (defined in appendix A)

$$
\left.\left(Q_{\dot{a}}+i \eta M_{\dot{a} \dot{b}} \tilde{Q}_{\dot{b}}\right) \|(r, s), \mathbf{y}_{t}\right\rangle=0,
$$

where $M_{\dot{a} \dot{b}}$ is given as in (1.4). Here $\eta= \pm 1$ distinguishes the $(r, s)$-brane from the $\overline{(r, s)}$ (the anti-brane). The gluing conditions for the fermions are uniquely determined by (2.1) and (2.3). However, the resulting conditions do not yet automatically imply that (2.3) actually holds. This constraint imposes additional restrictions on the set of $(r, s)$ branes, and may require a modification of the Neumann conditions (2.2), as will be described in section 1 .

By considering the commutator of (2.3) with $x_{0}^{I}$ it follows that the boundary state must be annihilated by

$$
\left.\left.\left(S_{0}^{a}+i \eta M_{a b} \tilde{S}_{0}^{b}\right) \|(r, s), \mathbf{y}_{t}\right\rangle\right)=0
$$

which implies that a complex combination of the kinematical supersymmetries is preserved by the boundary state. With this gluing condition it follows that the Dirichlet zero mode part of $Q_{\dot{a}}+i \eta(M \tilde{Q})_{\dot{a}}$ (i.e. the terms proportional to $x_{0}^{I}$ and $p_{0}^{I}$ for which $I$ is a Dirichlet direction) annihilates the boundary state for a class II brane irrespective of the transverse position $\mathbf{y}_{t}$. On the other hand, for class I branes, this condition is only satisfied if $y_{t}^{I}=0$ (unless we modify the gluing conditions (2.1) and (2.2)).

The gluing condition relating the non-zero modes $S_{n}^{a}$ and $\tilde{S}_{-n}^{b}$ at the boundary can be determined by first writing the non-zero mode part of the dynamical supercharge in (2.3) explicitly as

$$
\begin{aligned}
& \sum_{n=1}^{\infty}\left(c_{n} \gamma^{I}\left(\alpha_{-n}^{I} S_{n}+\alpha_{n}^{I} S_{-n}\right)+\frac{\eta m}{2 \omega_{n} c_{n}}\left(M \gamma^{I} \Pi\right)\left(\alpha_{-n}^{I} S_{n}-\alpha_{n}^{I} S_{-n}\right)\right) \\
& +i\left[\sum_{n=1}^{\infty}\left(\eta c_{n} M \gamma^{I}\left(\tilde{\alpha}_{-n}^{I} \tilde{S}_{n}+\tilde{\alpha}_{n}^{I} \tilde{S}_{-n}\right)+\frac{m}{2 \omega_{n} c_{n}}\left(\gamma^{I} \Pi\right)_{\dot{a} b}\left(\tilde{\alpha}_{-n}^{I} \tilde{S}_{n}^{b}-\tilde{\alpha}_{n}^{I} \tilde{S}_{-n}^{b}\right)\right)\right] .
\end{aligned}
$$

Using the fact that the boundary state is annihilated by $\alpha_{-n}^{I}-\tilde{\alpha}_{n}^{I}$ (for each Dirichlet direction $I$ ) this equation can be solved to determine the modes $\tilde{S}_{n}$ in terms of $S_{n}$. The solutions depend on the properties of $M$. However, by construction, these equations guarantee so far only that the Dirichlet part of the supercharges (i.e. the terms that are proportional to $\alpha_{n}^{I}$ or $\tilde{\alpha}_{n}^{I}$ with $I$ a Dirichlet direction) annihilate (2.3); whether the full equation (2.3) holds has to be analysed case by case.

Class I. In this case $\Pi M \Pi M=-1$, which is the condition assumed in [11, 9]. The solution of (2.3) is the same as in flat space,

$$
\left.\left(S_{n}^{a}+i \eta M_{a b} \tilde{S}_{-n}^{b}\right) \|(r, r+2), \mathbf{y}_{t}=0\right\rangle=0,
$$

which implies that the density of $\left(Q_{a}+i \eta(M \tilde{Q})_{a}\right)$ annihilates the boundary state. The cases with $(r+2, r)$ work in an equivalent fashion. With these gluing conditions it is then easy to see that the boundary state satisfies (2.3) with the standard gluing conditions for the Neumann directions (2.2). 
Class II. When $\Pi M \Pi M=+1$ the gluing conditions for the fermionic modes can again be determined as above, but now (2.3) is only satisfied provided that $(r, s)=(0,0) .^{3}$ Furthermore, if one relaxes the conditions (2.2) there are also solutions for the cases $(4,0)$ and $(0,4)$ which will be discussed in section 1 .

For the case of the $(0,0)$-brane, the matrix $M$ is simply $M=\mathbb{1}$, and the condition (2.3) can be expressed in the form

$$
\left.\left.\left[\left(\mathbb{1}+\frac{\eta m}{2 \omega_{n} c_{n}^{2}} \Pi\right)_{a b} S_{n}^{b}+i \eta\left(\mathbb{1}-\frac{\eta m}{2 \omega_{n} c_{n}^{2}} \Pi\right)_{a b} \tilde{S}_{-n}^{b}\right] \|(0,0), \mathbf{y}\right\rangle\right\rangle=0
$$

for all $n \in \mathbb{Z}, n \neq 0$. Note that since $\omega_{-n}=-\omega_{n}$ and $c_{-n}=c_{n}$, this formula gives the correct expression both for positive and negative $n$. This can be simplified using

$$
\left(\mathbb{1}+\frac{\eta m}{2 \omega_{n} c_{n}^{2}} \Pi\right)_{a b}\left(1-\frac{\eta m}{2 \omega_{n} c_{n}^{2}} \Pi\right)_{b c}=\frac{2 n}{m^{2}}\left(\omega_{n}-n\right) \delta_{a c},
$$

which leads to the conditions

$$
\left.\left(S_{n}^{a}+i \eta R_{n}^{a b} \tilde{S}_{-n}^{b}\right) \|(0,0), \mathbf{y}\right\rangle=0,
$$

where $R_{n}$ is the matrix

$$
R_{n}=\frac{1}{n}\left(\omega_{n} \mathbb{1}-\eta m \Pi\right) .
$$

It is worth noting that $R_{n}$ is not orthogonal, but that

$$
R_{n} R_{-n}^{T}=\mathbb{1} .
$$

This equation is required to make the above gluing conditions self-consistent.

The expression for $R_{n}$ can be simplified further by decomposing the $S O(8)$ spinors $S_{n}$ and $\tilde{S}_{n}$ into spinors of definite $S O(4)$ chiralities by defining

$$
S_{n}^{+}=\frac{1}{2}(1+\Pi) S_{n}, \quad S_{n}^{-}=\frac{1}{2}(1-\Pi) S_{n},
$$

so that $\Pi S_{n}^{ \pm}= \pm S_{n}^{ \pm}$, and similarly for $\tilde{S}_{n}$. Then (2.9) can be rewritten as

$$
\left.\left.\left(S_{n}^{ \pm}+i \eta R_{n}^{ \pm} \tilde{S}_{-n}^{ \pm}\right) \|(0,0), \mathbf{y}\right\rangle\right\rangle=0
$$

where

$$
R_{n}^{ \pm}=\frac{\omega_{n} \mp \eta m}{n}=\sqrt{\frac{\omega_{n} \mp \eta m}{\omega_{n} \pm \eta m}} .
$$

Given this expression, it is easy to write down the full boundary state

$$
\left.\|(0,0), \mathbf{y}, \eta\rangle=\mathcal{N}_{(0,0)} \exp \left(\sum_{k=1}^{\infty} \frac{1}{\omega_{k}} \alpha_{-k}^{I} \tilde{\alpha}_{-k}^{I}-i \eta R_{k}^{+} S_{-k}^{+} \tilde{S}_{-k}^{+}-i \eta R_{k}^{-} S_{-k}^{-} \tilde{S}_{-k}^{-}\right) \|(0,0)\right\rangle_{0},
$$

\footnotetext{
${ }^{3}$ Here we are assuming that the $D$-brane has at least one transverse Dirichlet direction; it is also possible to solve these equations for the case of the $(4,4)$-brane, but the resulting boundary state does not have a sensible flat space limit, and is probably inconsistent.
} 
where $\mathcal{N}_{(0,0)}$ is a normalisation constant that will turn out to equal

$$
\mathcal{N}_{(0,0)}=(4 \pi m)^{2}
$$

and the ground state component is

$$
\|(0,0)\rangle\rangle_{0}=(|I\rangle|I\rangle+i \eta|\dot{a}\rangle|\dot{a}\rangle) e^{-m \mathbf{y}^{2} / 2} e^{\frac{1}{2} a_{0}^{I} a_{0}^{I}-i \sqrt{2 m} y^{I} a_{0}^{I}}|0\rangle_{b}
$$

Here the first bracket describes the 'fermionic' part of the ground state, while the second part describes its 'bosonic'. This ground state is just the linear sum of the dilaton and $R R$ scalar that enters the flat-space boundary $D$-instanton state. This zero-mode part is an eigenstate of the closed-string hamiltonian, in contrast to the ground-state factors in the class I branes discussed in [9]. When $m \rightarrow 0$, the gluing condition (2.9) reduces to the usual flat-space result since $R_{n}=\mathbb{1}$ for $m=0$.

\subsection{Cylinder diagrams involving the $D$-instanton}

Given the explicit description for the $D$-instanton boundary state we can now evaluate the sum over cylindrical world-sheets with one boundary on a $(0,0)$-brane (or $D$-instanton) while the other boundary describes in turn a $(0,0)$-brane, a $\overline{(0,0)}$-brane (or anti $D$ instanton), or one of the class I branes discussed in [11, 9]. From the closed-string point of view that is considered in this section, this involves the overlap of two boundary states with non-zero $p^{+}$that are separated in the $x^{+}$direction by $X^{+}$, as well as the $x^{I}$ directions. The diagrams can also be identified with a trace over the states of the open strings joining the separated $D$-branes as will be seen in the next section. The proof that the closed-string construction gives the same expression as the open-string construction is a consequence of the modular property of the functions $g_{2}$ and $\hat{g}_{4}(1.18)$ that is explained in appendix B.

The cylinder diagram can be expressed as a closed-string propagator between the appropriate boundary states The overlap between a boundary state of a euclidean $D p$ brane located at a transverse position $y_{1}^{I}$ and a euclidean $D p^{\prime}$-brane at $y_{2}^{I}$ is given by [9],

$$
\mathcal{A}_{p^{\prime} \eta^{\prime} ; p \eta}\left(t ; \mathbf{y}_{\mathbf{1}}, \mathbf{y}_{\mathbf{2}}\right)=\left\langle\left\langle p^{\prime}, \mathbf{y}_{\mathbf{2}}, \eta^{\prime}\left\|e^{-2 \pi t H^{\text {closed }} p^{+}}\right\| p, \mathbf{y}_{\mathbf{1}}, \eta\right\rangle\right\rangle,
$$

where $t=X^{+} / 2 \pi p^{+}, H^{\text {closed }}$ is the closed-string light-cone gauge hamiltonian, and $\eta, \eta^{\prime}=$ + indicates that the boundary state describes an instanton, whereas $\eta, \eta^{\prime}=-$ indicates an anti-instanton. The overlap can be determined by standard methods (noting that the 'in'-state and the 'out'-state have opposite light-cone momentum $p^{+}$, and therefore $m$ takes the opposite value for the 'in'- and 'out'-state).

Let us begin by discussing the case of the overlap between two (0,0)-branes. As we have seen the fermionic ground state (2.17) is an eigenstate of the closed string light cone Hamiltonian, and therefore the overlap between two $(0,0)$-branes vanishes so that $\mathcal{A}_{(0,0) ;(0,0)}\left(t ; \mathbf{y}_{\mathbf{1}}, \mathbf{y}_{\mathbf{2}}\right)=0$. The same comment also applies to the overlaps between the $(0,0)$ and $(3,1)$ (or $(1,3))$ boundary states, $\mathcal{A}_{(0,0) ;(3,1)}\left(t ; \mathbf{y}_{\mathbf{1}}, \mathbf{y}_{\mathbf{2}}\right)=0$. Both of these results are direct consequences of the corresponding statements in the flat-space case [10]. 
Next consider the case of the overlap between a $(0,0)$ and a $\overline{(0,0)}$. Using the boundary state (2.15) and the fact that $R^{ \pm}(m, \eta) R^{ \pm}(-m,-\eta)=\left(R^{ \pm}(m, \eta)\right)^{2}$, together with standard oscillator algebra leads to the expression for the cylinder,

$$
\mathcal{A}_{\overline{(0,0)} ;(0,0)}\left(t ; \mathbf{y}_{\mathbf{1}}, \mathbf{y}_{\mathbf{2}}\right)=h_{0}\left(\mathbf{y}_{1}, \mathbf{y}_{2}\right) \frac{\left(g_{2}^{(m)}(t)\right)^{4}}{\left(f_{1}^{(m)}(t)\right)^{8}}
$$

where $f_{1}^{(m)}$ arises from each of the transverse integer-moded bosons (and is defined in (B.1), see [9]) and

$$
h_{0}\left(\mathbf{y}_{1}, \mathbf{y}_{2}\right)=\exp \left(-\frac{m\left(1+q^{m}\right)\left(\mathbf{y}_{1}^{2}+\mathbf{y}_{2}^{2}\right)}{2\left(1-q^{m}\right)}+\frac{2 m q^{\frac{m}{2}} \mathbf{y}_{1} \cdot \mathbf{y}_{2}}{\left(1-q^{m}\right)}\right) .
$$

Furthermore, each pair of fermions gives a factor

$$
g_{2}^{(m)}(t)=4 \pi m q^{-2 \Delta_{m}} q^{m / 2} \prod_{n=1}^{\infty}\left(1+\left(\frac{\omega_{n}+m}{\omega_{n}-m}\right) q^{\omega_{n}}\right)\left(1+\left(\frac{\omega_{n}-m}{\omega_{n}+m}\right) q^{\omega_{n}}\right),
$$

where the 'offset' $\Delta_{m}$ is the same offset that arises in the definition of $f_{1}^{(m)}$. In particular, the total offset of (2.19) is therefore $q^{2 m}$, in agreement with the fact that the lowest closed string state that couples to the boundary states is the fermionic ground state in (2.17) whose light-cone energy is $2 \mathrm{~m}$. (This state is characterised by the condition that is is annihilated (for $\eta=+1$, say) by $\theta_{L}^{a}$ and $\theta_{R}^{a}$.)

Similar arguments for the overlap between a $(0,0)$ and a $(0,2)$ at the origin lead to

$$
\mathcal{A}_{(0,0) ;(0,2)}(t ; \mathbf{y}, \mathbf{0})=2 \sinh (\pi m) j_{0}(\mathbf{y}) \frac{\left(g_{2}^{(m)}\left(t^{2}\right)\right)^{2}}{\left(f_{1}^{(m)}(t)\right)^{6}\left(f_{2}^{(m)}(t)\right)^{2}},
$$

where the prefactor of $2 \sinh (\pi m)$ comes from the normalisation of the $(0,2)$ boundary state that was determined in [9], and $\mathbf{y}$ describes the position of the $(0,0)$ (the $(0,2)$ is assumed to be located at the origin in the transverse space). Furthermore

$$
j_{0}(\mathbf{y})=\exp \left(-\frac{m\left(1+q^{m}\right) \mathbf{y}_{t}^{2}}{2\left(1-q^{m}\right)}\right) \exp \left(-\frac{m\left(1-q^{m}\right) \mathbf{y}_{l}^{2}}{2\left(1+q^{m}\right)}\right),
$$

where $\mathbf{y}_{t}$ is the component of $\mathbf{y}$ in the directions transverse to the $(0,2)$, while $\mathbf{y}_{l}$ denotes the components of $\mathbf{y}$ along the world-volume directions of the $(0,2)$.

Similarly, the overlap of a $(0,0)$ with a class I $(2,4)$-brane (a euclidean $D 5$-brane) is

$$
\mathcal{A}_{(0,0) ;(2,4)}(t ; \mathbf{y}, \mathbf{0})=\frac{j_{0}(\mathbf{y})}{2 \sinh (m \pi)} \frac{\left(g_{2}^{(m)}\left(t^{2}\right)\right)^{2}}{\left(f_{1}^{(m)}(t)\right)^{2}\left(f_{2}^{(m)}(t)\right)^{6}},
$$

where now $\mathbf{y}_{t}$ is the component of $\mathbf{y}$ in the directions transverse to the $(2,4)$-brane, while $\mathbf{y}_{l}$ denotes the components of $\mathbf{y}$ along the world-volume directions of the $(2,4)$. 


\section{The open string point of view}

In this section the $(0,0)$ (D-instanton) is analysed from the open string point of view. It follows from the equations of motion [12] that the open string functions satisfy the equations

$$
\begin{aligned}
& \partial_{+} S(\sigma, \tau)=\widehat{m} \Pi \tilde{S}(\sigma, \tau), \\
& \partial_{-} \tilde{S}(\sigma, \tau)=-\widehat{m} \Pi S(\sigma, \tau),
\end{aligned}
$$

where $\widehat{m}=\mu x^{+}$is the mass parameter in the open string light cone gauge for which both light cone directions satisfy Dirichlet boundary conditions [9]. The general solution can be written as 14

$$
\begin{array}{r}
S(\sigma, \tau)=S_{0}^{\prime} \cos (\widehat{m} \tau)+\Pi \tilde{S}_{0}^{\prime} \sin (\widehat{m} \tau)+S_{0} \cosh (\widehat{m} \sigma)+\Pi \tilde{S}_{0} \sinh (\widehat{m} \sigma) \\
+\sum_{n \neq 0} c_{n}\left[S_{n} e^{-i\left(\widehat{\omega}_{n} \tau-n \sigma\right)}+\frac{i}{\widehat{m}}\left(\widehat{\omega}_{n}-n\right) \Pi \tilde{S}_{n} e^{-i\left(\widehat{\omega}_{n} \tau+n \sigma\right)}\right], \\
\tilde{S}(\sigma, \tau)=-\Pi S_{0}^{\prime} \sin (\widehat{m} \tau)+\tilde{S}_{0}^{\prime} \cos (\widehat{m} \tau)+\tilde{S}_{0} \cosh (\widehat{m} \sigma)+\Pi S_{0} \sinh (\widehat{m} \sigma) \\
+\sum_{n \neq 0} c_{n}\left[\tilde{S}_{n} e^{-i\left(\widehat{\omega}_{n} \tau+n \sigma\right)}-\frac{i}{\widehat{m}}\left(\widehat{\omega}_{n}-n\right) \Pi S_{n} e^{-i\left(\widehat{\omega}_{n} \tau-n \sigma\right)}\right],
\end{array}
$$

where $\widehat{\omega}_{n}=\sqrt{\widehat{m}^{2}+n^{2}}$.

\subsection{Boundary conditions and mode expansions}

The boundary conditions on the open string determine the mode expansion. The nature of this expansion depends sensitively on the particular branes on which the open string terminates and we will discuss them on a case by case basis.

3.1.1 $(0,0)-(0,0)$

In the case of the open string between a $(0,0)$-brane at $\mathbf{y}_{1}$, and a $(0,0)$-brane at $\mathbf{y}_{2}$ the open-string bosonic coordinates have the mode expansion

$$
\begin{aligned}
x^{I} & =y_{1}^{I} \cosh (\widehat{m} \sigma)+\frac{y_{2}^{I}-y_{1}^{I} \cosh (\widehat{m} \pi)}{\sinh (\widehat{m} \pi)} \sinh (\widehat{m} \sigma)+\sum_{l \neq 0} \frac{2}{\widehat{\omega}_{l}} \alpha_{l}^{I} e^{-i \widehat{\omega}_{l} \tau} \sin (l \sigma), \\
\mathcal{P}^{I} & =-2 i \sum_{l \neq 0} \alpha_{l}^{I} e^{-i \widehat{\omega}_{l} \tau} \sin (l \sigma), \\
x^{I} & =\widehat{m} y_{1}^{I} \sinh (\widehat{m} \sigma)+\widehat{m} \frac{y_{2}^{I}-y_{1}^{I} \cosh (\widehat{m} \pi)}{\sinh (\widehat{m} \pi)} \cosh (\widehat{m} \sigma)+2 \sum_{l \neq 0} \frac{l}{\widehat{\omega}_{l}} \alpha_{l}^{I} e^{-i \widehat{\omega}_{l} \tau} \cos (l \sigma) .
\end{aligned}
$$

On the other hand the fermionic modes are restricted by the boundary conditions

$$
S(\sigma, \tau)=\tilde{S}(\sigma, \tau) \quad \text { for } \sigma=0, \pi .
$$

For the zero-modes this condition requires that $S_{0}^{\prime}=\tilde{S}_{0}^{\prime}=0$ and that the second set of zero modes are related,

$$
S_{0}=\tilde{S}_{0}
$$


Furthermore, (3.6) implies that the non-zero fermion modes of (3.3) are related by

$$
\tilde{S}_{n}=T_{n} S_{n}
$$

where $n \in \mathbb{Z}$ with $n \neq 0$ and

$$
T_{n}=\frac{1}{\widehat{\omega}_{n}}[n \mathbb{1}+i \widehat{m} \Pi] .
$$

The matrix $T_{n}$ is unitary, $T_{n} T_{n}^{*}=\mathbb{1}$.

We can now discuss the open-string supercharges. The mode expansions of $S$ and $\tilde{S}$, together with (3.8) lead to the expansions

$$
\begin{aligned}
(S-\tilde{S})= & 2 \sum_{n \neq 0} c_{n} \sin (n \sigma)\left(i \mathbb{1}-\frac{\left(\widehat{\omega}_{n}-n\right)}{\widehat{m}} \Pi\right) S_{n} e^{-i \widehat{\omega}_{n} \tau}, \\
(S+\tilde{S})= & 2 S_{0} \cosh (\widehat{m} \sigma)+2 \Pi S_{0} \sinh (\widehat{m} \sigma) \\
& +2 \sum_{n \neq 0} c_{n}\left[\cos (n \sigma)\left(\frac{n}{\widehat{\omega}_{n}}+i \frac{n\left(\widehat{\omega}_{n}-n\right)}{\widehat{m} \widehat{\omega}_{n}} \Pi\right) S_{n}\right. \\
& \left.+i \sin (n \sigma)\left(\frac{\left(\widehat{\omega}_{n}-n\right)}{\widehat{\omega}_{n}}-i \frac{\widehat{m}}{\widehat{\omega}_{n}} \Pi\right) S_{n}\right] e^{-i \widehat{\omega}_{n} \tau} .
\end{aligned}
$$

The combination $S(\sigma)+\tilde{S}(\sigma)$ is proportional to the density of the kinematic supercharge. Since this contains a term proportional to $\sum_{n \neq 0} \sin (n \sigma) c_{n} M_{n} S_{n} e^{-i \widehat{\omega}_{n} \tau}$, the supercharge, which is the integral of this density, has a complicated time dependence and is not conserved. ${ }^{4}$

The dynamical supercharge of this open superstring is given by the difference of the left and right moving supercharges. In flat space this can be seen by a careful analysis of T-duality from the open string on the D9-brane. Using the formulae given in [2] one has

$$
\mathcal{Q}=\frac{1}{2 \sqrt{X^{+}}} \int_{0}^{\pi} d \sigma\left[\mathcal{P}^{I} \gamma^{I}(\tilde{S}-S)+x^{I} \gamma^{I}(S+\tilde{S})+\widehat{m} x^{I} \gamma^{I} \Pi(S+\tilde{S})\right] .
$$

This is time independent (and therefore conserved) as is shown in appendix C.2, where it is found to have the mode expansion

$$
\begin{array}{r}
\mathcal{Q}=\frac{2}{\sqrt{X^{+}}}\left[y_{2}^{I} \gamma^{I}\left(\cosh (\pi \widehat{m}) S_{0}+\sinh (\pi \widehat{m}) \Pi S_{0}\right)-y_{1}^{I} \gamma^{I} S_{0}\right. \\
\left.+\pi \sum_{n \neq 0} c_{n} \alpha_{n}^{I} \gamma^{I}\left(\mathbb{1}-i \frac{\left(\widehat{\omega}_{n}-n\right)}{\widehat{m}} \Pi\right) S_{-n}\right] .
\end{array}
$$

The anti-commutation relations for the fermionic modes are also determined in appendix C.1 and are given by

$$
\begin{aligned}
\left\{S_{n}^{a}, S_{m}^{b}\right\} & =\delta^{a b} \delta_{n,-m} \quad \text { if } n \neq 0 \text { or } m \neq 0, \\
\left\{S_{0}^{a}, S_{0}^{b}\right\} & =\frac{\pi \widehat{m}}{2 \sinh (\pi \widehat{m})}\left(\cosh (\pi \widehat{m}) \delta^{a, b}-\sinh (\pi \widehat{m}) \Pi^{a, b}\right) .
\end{aligned}
$$

\footnotetext{
${ }^{4}$ The 'conserved' kinematical supercharges for the class I branes are not strictly speaking independent of $\tau$. However, their $\tau$ dependence is simply a consequence of the commutation relations of the fermionic zero modes with the hamiltonian (A.19.
} 
It is easy to calculate the anti-commutator of the supercharges $\mathcal{Q}_{\dot{a}}$, and one finds

$$
\left\{\mathcal{Q}_{\dot{a}}, \mathcal{Q}_{\dot{b}}\right\}=2 \delta_{\dot{a} \dot{b}} H^{\text {open }}+\frac{2 \pi \widehat{m}}{X^{+}}\left(\gamma^{I} \Pi \gamma^{J}\right)_{\dot{a} \dot{b}}\left(y_{2}^{I} y_{2}^{J}-y_{1}^{I} y_{1}^{J}\right)
$$

where the open string Hamiltonian is defined by

$\frac{X^{+}}{2 \pi} H^{\text {open }}=\frac{\widehat{m}}{2 \sinh (\pi \widehat{m})}\left(\cosh (\pi \widehat{m})\left(y_{2}^{I} y_{2}^{I}+y_{1}^{I} y_{1}^{I}\right)-2 y_{2}^{I} y_{1}^{I}\right)+2 \pi \sum_{n>0}\left(\alpha_{-n}^{I} \alpha_{n}^{I}+\widehat{\omega}_{n} S_{-n}^{a} S_{n}^{a}\right)$,

and $n \in \mathbb{Z}$. The second term in (3.15) describes a central charge since it commutes with the supercharges, as well as the rotation generators in $S O(4) \times S O(4)$. It is important that there is no mass term for the fermionic zero modes in this hamiltonian.

Although the expression (3.16) has been obtained for the open string joining two $(0,0)$-branes it has the same structure for strings joining any pair of class II branes. In particular, there is no mass term for the fermionic zero modes. Recall that in the case of the hamiltonian of the class I $D$-branes the mass term has the form $S_{0} M \Pi S_{0}$ but such a term vanishes identically for any of the class II $D$-branes.

3.1.2 $(0,0)-\overline{(0,0)}$

Now consider the open string with one end on the $(0,0)$ and the other on the $\overline{(0,0)}$ (anti $D$-instanton). The bosons are still described by (3.5), but now the boundary conditions for the fermions, replacing (3.6), is

$$
S(0, \tau)=\tilde{S}(0, \tau) \quad S(\pi, \tau)=-\tilde{S}(\pi, \tau) .
$$

This boundary condition is incompatible with the presence of zero modes, which are therefore absent. The boundary condition at $\sigma=0$, requires that the non-zero modes have to be related as in (3.8), while the condition at $\sigma=\pi$ implies that

$$
\tilde{S}_{n}=-e^{2 \pi i n} T_{n}^{*} S_{n}, \quad \text { for } n \neq 0 .
$$

In order to construct a simultaneous solution to (3.8) and (3.18) let us write these equations in terms of the plus-minus components introduced before in (2.12). Then (3.8) becomes

$$
\tilde{S}_{n}^{ \pm}=T_{n}^{ \pm} S_{n}^{ \pm}, \quad \text { where } \quad T_{n}^{ \pm}=\frac{(n \pm i \widehat{m})}{\widehat{\omega}_{n}},
$$

while (3.18) is

$$
\tilde{S}_{n}^{ \pm}=-e^{2 \pi i n}\left(T_{n}^{ \pm}\right)^{*} S_{n}^{ \pm},
$$

and again both equations only hold for $n \neq 0$. It then follows that there exists a nontrivial solution provided that the mode number $n$ of $S_{n}^{+}$and $\tilde{S}_{n}^{+}$satisfies the transcendental equation

$$
n \in \mathcal{P}_{+}: \frac{n+i \widehat{m}}{n-i \widehat{m}}=-e^{2 \pi i n},
$$

while the corresponding condition for the mode number of $S_{n}^{-}$and $\tilde{S}_{n}^{-}$is

$$
n \in \mathcal{P}_{-}: \frac{n-i \widehat{m}}{n+i \widehat{m}}=-e^{2 \pi i n} .
$$


Both of these spaces of solutions contain a trivial zero mode solution with $n=0$, but we have seen that this is not an allowed mode of the open string. It is obvious that there are infinitely many other solutions for $n$ in both cases. For small $\widehat{m}$, the solutions to both equations are close to all the half-odd integers. Also, if $n \in \mathcal{P}_{+}$, i.e. if $n$ is a solution to the first equation, then $-n \in \mathcal{P}_{+}$, and similarly for $\mathcal{P}_{-}$. The hamiltonian for this open string is again given by (3.16), except that in the second sum $n \in \mathcal{P}_{ \pm}$.

\subsection{3 $(0,0)-(r, r+2)$}

We may also analyse the modes of an open string stretching between a $(0,0)$ and one of the $(r, r+2)$-branes (or class I branes) of [11, 9]. Let us first discuss the bosonic modes. For the directions that satisfy a Dirichlet boundary condition at both end-points (i.e. the DD directions), the bosons are still described by (3.5), and their contribution to the hamiltonian is given by the first term in (3.16). For the DN directions, the hamiltonian also depends on the position of the $(0,0)$. This is a consequence of the fact, explained in [9], that $x^{-}$ depends on the position on the world-volume of the class I branes. Taking into account the Wick rotation for $x^{+}$that is described in [9], it follows that the relevant contribution to the open string hamiltonian is given by

$$
H_{0}^{\text {open }}=\frac{\widehat{m} \tanh (\widehat{m} \pi)}{2} \mathbf{y}_{l}^{2} .
$$

In order to describe the fermions let us assume the $(r, r+2)$-brane is at $\sigma=0$, while the $(0,0)$ is at $\sigma=\pi$. The former boundary condition $(S(0, \tau)=M \tilde{S}(0, \tau))$ then leads to

$$
S_{n}=M \tilde{S}_{n}, \quad n \neq 0
$$

as well as

$$
S_{0}=M \tilde{S}_{0}, \quad S_{0}^{\prime}=M \tilde{S}_{0}^{\prime},
$$

where $-\mathbb{1}=\Pi M \Pi M$ [12]. At $\sigma=\pi$ the relevant boundary condition is $S(\pi, \tau)=\tilde{S}(\pi, \tau)$; this requires that the condition

$$
\tilde{S}_{n}=e^{2 \pi i n} T_{n} S_{n}, \quad n \neq 0
$$

is satisfied, as well as

$$
S_{0}=\tilde{S}_{0}, \quad S_{0}^{\prime}=\tilde{S}_{0}^{\prime}=0 .
$$

As before (3.26) can be rewritten as

$$
\tilde{S}_{n}^{ \pm}=e^{2 \pi i n} T_{n}^{ \pm} S_{n}^{ \pm}, \quad n \neq 0 .
$$

The open string modings now depend sensitively on the symmetry of $M$ :

Case (i) $M$ is symmetric and anti-commutes with $\Pi$. This is the case for the $(1,3)$ and (3,1)-brane where, for example, $M=\gamma^{1} \gamma^{2} \gamma^{3} \gamma^{5}$. Since $M^{2}=11$ half of the zero modes $S_{0}$ (namely those that have eigenvalue +1 under the action of $M$ ) satisfy both (3.25) and (3.27). The open string therefore contains four fermionic zero modes (that commute with 
the light cone hamiltonian). As regards the non-zero modes, (3.24) can be rewritten in terms of the \pm components as

$$
\left(\begin{array}{c}
S_{n}^{+} \\
S_{n}^{-}
\end{array}\right)=\left(\begin{array}{cc}
0 & M \\
M^{T} & 0
\end{array}\right)\left(\begin{array}{c}
\tilde{S}_{n}^{+} \\
\tilde{S}_{n}^{-}
\end{array}\right) .
$$

Combining this equation with $(3.28)$ gives

$$
\left(\begin{array}{c}
S_{n}^{+} \\
S_{n}^{-}
\end{array}\right)=e^{2 \pi i n}\left(\begin{array}{cc}
0 & M T_{n}^{-} \\
M^{T} T_{n}^{+} & 0
\end{array}\right)\left(\begin{array}{l}
S_{n}^{+} \\
S_{n}^{-}
\end{array}\right) .
$$

The condition for a consistent boundary condition is therefore

$$
S_{n}^{+}=e^{4 \pi i n} T_{n}^{-} T_{n}^{+} M M^{T} S_{n}^{+} .
$$

Since $T_{n}^{+} T_{n}^{-}=1$, this is the same condition as in flat space, and thus the modings of these open strings are exactly as in the flat space case. Since the number of DD and ND directions of the $(3,1)$-brane are both equal to four, four of the eight fermions are integer moded while the other four are half-integer moded.

Case (ii) $M$ is anti-symmetric and commutes with $\Pi$ which is the case for the $(2,4),(4,2)$, $(2,0)$ and $(0,2)$. One example is the D1-brane with $M=\gamma^{1} \gamma^{2}$. In this case, $M^{2}=-1$, and therefore none of the zero modes $S_{0}$ has eigenvalue +1 under the action of $M$. In particular, it therefore follows that there are no fermionic zero modes in this open string.

As regards the non-zero modes, (3.24) can be rewritten as

$$
\left(\begin{array}{c}
S_{n}^{+} \\
S_{n}^{-}
\end{array}\right)=\left(\begin{array}{cc}
M^{+} & 0 \\
0 & M^{-}
\end{array}\right)\left(\begin{array}{c}
\tilde{S}_{n}^{+} \\
\tilde{S}_{n}^{-}
\end{array}\right) .
$$

The eigenvalues of $M^{ \pm}$are $+i,+i,-i,-i$. Let us consider one of the plus-components $S_{n}^{+}$ (the analysis for the minus components is analogous). The conditions obtained from (3.24) and (3.28) then become

$$
1= \pm i e^{2 \pi i n} \frac{1}{\widehat{\omega}_{n}}(n+i \widehat{m}),
$$

where the sign on the right hand side depends on whether the eigenvalue under $M^{+}$is $\pm i$. Squaring this identity gives

$$
-1=e^{4 \pi i n} \frac{n+i \widehat{m}}{n-i \widehat{m}}=e^{2 \pi i(2 n)} \frac{(2 n)+i(2 \widehat{m})}{(2 n)-i(2 \widehat{m})} .
$$

Thus for each pair of $S_{n}^{+}$modes with eigenvalues $\pm i$, the mode numbers must satisfy $2 n \in \mathcal{P}_{+}^{(2 \widehat{m})}$. Similarly, for each pair of $S_{n}^{-}$modes with eigenvalues $\pm i$, the mode numbers must satisfy $2 n \in \mathcal{P}_{-}^{(2 \widehat{m})}$.

\subsection{Consistency of one-loop open-string amplitudes}

Given the explicit knowledge of the open string spectra involving the $D$-instanton boundary condition, we can now also evaluate the cylinder diagram as a trace over the open string states, giving [9]

$$
Z_{p_{1} ; p_{2}}(\tilde{t})=\operatorname{tr} e^{-\frac{X^{+}}{2 \pi} H^{\text {open }} \tilde{t}}
$$


where $\tilde{t}=1 / t=2 \pi p^{+} / X^{+}$and $H^{o p e n}$ is the open-string hamiltonian in the light-cone gauge. The resulting expressions must agree with what was obtained in the previous section from a closed-string point of view. This is the open-closed consistency condition that will be checked in the following.

The open string with $(0,0)$-brane boundary conditions at both ends, has eight integermoded world-sheet bosons and eight integer-moded world-sheet fermions. Furthermore, the fermionic zero modes commute with the Hamiltonian, and the trace therefore vanishes, in agreement with the closed string result.

The analysis is similar for the open string with a $(0,0)$ boundary condition at one end, and a $(1,3)$ boundary condition at the other. Again, there are four fermionic zero modes that commute with the open string hamiltonian, and thus the cylinder diagram vanishes, in agreement with the closed string result.

The situation is more interesting for the case of the open string with one boundary on the $(0,0)$, and the other on the $\overline{(0,0)}$. Evaluating the trace now gives an expression of the form

$$
Z_{(0,0) ; \overline{(0,0)}}=\hat{h}_{0}\left(\mathbf{y}_{1}, \mathbf{y}_{2}\right) \frac{\left(\widehat{g}_{4}^{(\widehat{m})}(\tilde{t})\right)^{4}}{\left(f_{1}^{(\widehat{m})}(\tilde{t})\right)^{8}}
$$

where $\hat{h}_{0}\left(\mathbf{y}_{1}, \mathbf{y}_{2}\right)$ describes the contribution from the first term in (3.16)

$$
\hat{h}_{0}\left(\mathbf{y}_{1}, \mathbf{y}_{2}\right)=\exp \left(-\frac{\widehat{m} \tilde{t}}{2 \sinh (\pi \widehat{m})}\left[\cosh (\pi \widehat{m})\left(\mathbf{y}_{1}{ }^{2}+\mathbf{y}_{2}^{2}\right)-2 \mathbf{y}_{1} \cdot \mathbf{y}_{2}\right]\right),
$$

and the function $\widehat{g}_{4}^{(\widehat{m})}(\tilde{t})$ is defined by

$$
\widehat{g}_{4}^{(\widehat{m})}(\tilde{t})=\tilde{q}^{-\widehat{\Delta}_{\hat{m}}} \prod_{l \in \mathcal{P}_{+}}\left(1-\tilde{q}^{\widehat{\omega}_{l}}\right)^{\frac{1}{2}} \prod_{l \in \mathcal{P}_{-}}\left(1-\tilde{q}^{\widehat{\omega}_{l}}\right)^{\frac{1}{2}} .
$$

The products in (3.38) are over all the values of $l$ that satisfy (1.16) and (1.17), respectively. In both cases, the value $l=0$ is included in the product. The total $l=0$ contribution $\left(1-\tilde{q}^{\widehat{m}}\right)^{4}$ cancels the zero mode contribution from $\left(f_{1}^{(\widehat{m})}(\tilde{t})\right)^{8}$. This is in agreement with the spectrum since the contribution of the bosonic zero modes is already described by the prefactor $\hat{h}_{0}\left(\mathbf{y}_{1}, \mathbf{y}_{2}\right)$. The off-set $\widehat{\Delta}_{\widehat{m}}$ is defined in (B.15) of appendix B.

Using the relations $q^{m}=e^{-2 \pi t m}=e^{-2 \pi \widehat{m}}$, as well as $m=\widehat{m} \tilde{t}$, it is easy to see that $h_{0}\left(\mathbf{y}_{1}, \mathbf{y}_{2}\right)=\hat{h}_{0}\left(\mathbf{y}_{1}, \mathbf{y}_{2}\right)$. Furthermore, as is shown in appendix D, the functions $g_{2}$ and $\widehat{g}_{4}$ satisfy the non-trivial identity

$$
g_{2}^{(m)}(t)=\widehat{g}_{4}^{(\widehat{m})}(\tilde{t})
$$

This implies that

$$
\mathcal{A}_{p_{1} ; p_{2}}(t)=Z_{p_{1} ; p_{2}}(\tilde{t})
$$

and thus that the two calculations agree, as they should.

Similarly, the one-loop contribution of an open string between the $(0,0)$ and the $(2,0)$ is

$$
Z_{(0,0) ;(2,0)}=\hat{\jmath}_{0}(\mathbf{y}) \tilde{q}^{-\frac{\hat{m}}{2}}\left(1-\tilde{q}^{\widehat{m}}\right) \frac{\left(\widehat{g}_{4}^{(2 \widehat{m})}\left(\tilde{t}^{1 / 2}\right)\right)^{2}}{\left(f_{1}^{(\widehat{m})}(\tilde{t})\right)^{6}\left(f_{4}^{(\widehat{m})}(\tilde{t})\right)^{2}},
$$


where the prefactor of $\tilde{q}^{-\widehat{m} / 2}\left(1-\tilde{q}^{\widehat{m}}\right)$ cancels the part of the zero mode contribution from $\left(f_{1}^{(\widehat{m})}(\tilde{t})\right)^{6}$ that is not cancelled by $\left(\widehat{g}_{4}^{(2 \widehat{m})}\left(\tilde{t}^{1 / 2}\right)\right)^{2}$, and

$$
\hat{\jmath}_{0}(\mathbf{y})=\exp \left(-\frac{\widehat{m} \tilde{t}}{2}\left[\mathbf{y}_{t}^{2} \frac{\cosh (\pi \widehat{m})}{\sinh (\pi \widehat{m})}+\mathbf{y}_{l}^{2} \frac{\sinh (\pi \widehat{m})}{\cosh (\pi \widehat{m})}\right]\right) .
$$

Again, it is easy to see that $j_{0}(\mathbf{y})=\hat{\jmath}_{0}(\mathbf{y})$, and that $(3.40)$ is again a consequence of (3.39). Finally, the result for the case of the open string between the $(0,0)$ and the $(4,2)$-brane is

$$
Z_{(0,0) ;(4,2)}=\hat{\jmath}_{0}(\mathbf{y}) \tilde{q}^{\frac{\hat{m}}{2}}\left(1-\tilde{q}^{\widehat{m}^{2}}\right)^{-1} \frac{\left(\widehat{g}_{4}^{(2 \widehat{m})}\left(\tilde{t}^{1 / 2}\right)\right)^{2}}{\left(f_{1}^{(\widehat{m})}(\tilde{t})\right)^{2}\left(f_{4}^{(\widehat{m})}(\tilde{t})\right)^{6}}
$$

and the consistency of the closed-string and open-string sectors follows by the same arguments as before.

\section{4. $(4,0)$ and $(0,4)$ with flux}

The (4,0)-brane and the (0,4)-brane couple to the self-dual background $R R$ four-form potential. This means that a nontrivial Born-Infeld flux is necessarily switched on in the world-volume. In turn, this affects the Neumann boundary conditions. We will determine the open-string boundary conditions by enforcing the consistency of the cylinder diagrams under the $S$ modular transformation, starting from the closed-string boundary states.

For definiteness, let us consider the (4,0)-brane (the construction for the (0,4)-brane is similar). The corresponding boundary state should be characterised by the gluing conditions

$$
\left.\left.\left(Q_{\dot{a}}+i \eta \Pi_{\dot{a} \dot{b}} \tilde{Q}_{\dot{b}}\right) \|(4,0), \eta\right\rangle\right)=0 .
$$

In terms of the chiral \pm components this is the condition

$$
\left.\left.\left(Q_{\dot{a}}^{ \pm} \pm i \eta \tilde{Q}_{\dot{a}}^{ \pm}\right) \|(4,0), \eta\right\rangle\right\rangle=0
$$

In addition to (4.2), the boundary state should satisfy the Dirichlet gluing conditions

$$
\begin{aligned}
\left.\left(\alpha_{k}^{i^{\prime}}-\tilde{\alpha}_{-k}^{i^{\prime}}\right) \|(4,0), \eta\right\rangle & =0, \quad k \in \mathbb{Z} \backslash\{0\}, \\
\left.\left(\bar{a}_{0}^{i^{\prime}}-a_{0}^{i^{\prime}}+i \sqrt{2 m} y_{t}^{i^{\prime}}\right) \|(4,0), \eta\right\rangle & =0,
\end{aligned}
$$

where $i^{\prime}$ denotes the coordinates that are transverse to the $(4,0)$ and $y_{t}^{i^{\prime}}$ is the position of the $(4,0)$ in these directions. As before, (4.2) together with (4.3) already determines the gluing conditions for all fermionic modes. Once these have been determined they will imply, using (4.2), what the gluing conditions for the bosonic modes along the world-volume of the $(4,0)$ must be.

From the condition that $(Q+i \eta \tilde{Q})^{+}$should annihilate the boundary state, using (4.3), we find that

$$
\left.\left.\left(S_{n}^{+}+i \eta \frac{\omega_{n}-m \eta}{n} \tilde{S}_{-n}^{+}\right) \|(4,0), \eta\right\rangle\right)=0, \quad n \neq 0
$$


while the condition that $(Q-i \eta \tilde{Q})^{-}$annihilates the boundary state gives

$$
\left.\left.\left(S_{n}^{-}-i \eta \frac{\omega_{n}-m \eta}{n} \tilde{S}_{-n}^{-}\right) \|(4,0), \eta\right\rangle\right\rangle=0, \quad n \neq 0 .
$$

Both of these identities follow directly from the analysis described before for the $(0,0)$ brane. With these fermionic gluing conditions, the terms in (4.2) proportional to $\alpha_{n}^{i^{\prime}}$ annihilate the boundary state. On the other hand, the terms that are proportional to $\alpha_{n}^{i}$ vanish if and only if

$$
\left.\left.\left[\alpha_{n}^{i}+\left(\frac{\omega_{n}-m \eta}{\omega_{n}+m \eta}\right) \tilde{\alpha}_{-n}^{i}\right] \|(4,0), \eta\right\rangle\right\rangle=0
$$

for all $n \neq 0$. This condition reduces to the standard Neumann boundary condition for $m \rightarrow 0$.

The zero-mode component of (4.2) requires, using the second equation of (4.3), that

$$
\left.\left(S_{0}^{a}+i \eta \Pi_{a b} \tilde{S}_{0}^{b}\right) \|(4,0), \eta\right\rangle=0 .
$$

Furthermore, the bosonic zero-mode condition for the transverse directions is

$$
\left(p_{0}^{i}-i \eta m x_{0}^{i}\right) \|(4,0), \eta \gg=0 .
$$

So the complete set of gluing conditions for the supersymmetric $(4,0)$-brane with flux is given by (4.3)-(4.8). The bosonic gluing conditions can be summarised as

$$
\left.\left.\left(\mathcal{P}^{i}-i \eta m x^{i}\right)\right|_{\tau=0} \|(4,0), \eta\right\rangle=0 .
$$

A notable feature of condition (4.8) is that for $\eta=+$ the bosonic zero mode ground state is the Fock space ground state since it is annihilated by $\bar{a}^{i}$. On the other hand, the ground state for the anti-brane $(\eta=-)$ would be the state that is killed by the zero mode creation operator, $a^{i}$. This would mean that the anti-brane had to lie in a different Fock space that decouples from all of the other branes. The conclusion is therefore that there is no supersymmetric anti-brane for the $(4,0)$ (and $(0,4))$ cases. Which of the two, the brane or the anti-brane, is supersymmetric obviously depends on the sign of $m$, and thus on the sign of the $R R$ background flux.

\subsection{The open string description}

In order to deduce the open-string description of this boundary condition, let us analyse one of the non-vanishing overlaps involving the (4,0)-brane. To be specific, consider its overlap with the $(2,0)$-brane, where, for simplicity, both branes are taken to be at the origin in the transverse space. Using the same arguments as before, one finds that this overlap is

$$
\mathcal{A}_{(2,0) ;(4,0)}(t)=(2 \sinh (\pi m))^{2} \frac{\left(g_{1,-}^{(m)}(2 t)\right)^{2}\left(g_{2,-}^{(m)}(2 t)\right)^{2}}{\left(f_{1}^{(m)}(t)\right)^{4}\left(g_{1,-}^{(m)}(t)\right)^{2}\left(g_{2,-}^{(m)}(t)\right)^{2}},
$$


where the functions $g_{1,-}^{(m)}(t)$ and $g_{2,-}^{(m)}(t)$ and their transformation properties are defined in appendix B.1. Using the formulae given there, the relevant open string has a one-loop partition function given by

$$
Z_{(2,0) ;(4,0)}(\tilde{t})=\tilde{q}^{-\widehat{m}}\left(1-\tilde{q}^{\widehat{m}}\right)^{2} \frac{\left(\hat{g}_{1,+}^{(2 \widehat{m})}(\tilde{t} / 2)\right)^{2}\left(\hat{g}_{4,+}^{(2 \widehat{m})}(\tilde{t} / 2)\right)^{2}}{\left(f_{1}^{(\widehat{m})}(\tilde{t})\right)^{4}\left(\hat{g}_{1,+}^{(\widehat{m})}(\tilde{t})\right)^{2}\left(\hat{g}_{4,+}^{(\widehat{m})}(\tilde{t})\right)^{2}} .
$$

This one-loop amplitude is consistent with the boundary condition for the (4,0)-brane

$$
\tilde{S}_{n}^{ \pm}= \pm \frac{n+i \widehat{m}}{\widehat{\omega}_{n}} S_{n}^{ \pm}
$$

as well as

$$
\alpha_{n}^{i}=\frac{n-i \widehat{m}}{n+i \widehat{m}} \tilde{\alpha}_{n}^{i}, \quad \alpha_{n}^{i^{\prime}}=-\tilde{\alpha}_{n}^{i^{\prime}}
$$

The bosonic relations are equivalent to the condition that

$$
\begin{aligned}
x^{\prime i}(\sigma, \tau)-\widehat{m} x^{i}(\sigma, \tau) & =0, \\
x^{i^{\prime}}(\sigma, \tau) & =y^{i^{\prime}}
\end{aligned}
$$

at the boundary corresponding to the $(4,0)$ brane, while the fermionic conditions are equivalent to

$$
S(\sigma, \tau)=\Pi \tilde{S}(\sigma, \tau)
$$

In section 5 we will point out that the modified bosonic conditions (4.14) also arise in the context of the $(+,-; 4,0)$-brane as described in [7, 8]. As in the case of the $(0,0)-(0,0)$ system the cylinder connecting two $(4,0)$-branes vanishes, $\mathcal{A}_{(4,0) ;(4,0)}(t)=0$.

\subsection{Supersymmetry}

Having determined the boundary conditions for the (4,0)-brane, we can now deduce the mode expansion of the bosonic and fermionic fields for the open string both of whose ends lies on a $(4,0)$-brane. The components of $S^{+}$and $\tilde{S}^{+}$have the mode expansions given in (3.10) while the components of $S^{-}$and $\tilde{S}^{-}$have the same mode expansion as the components of $S^{-}$and $\tilde{S}^{-}$for the $\overline{(0,0)}$. Thus,

$$
\begin{aligned}
(S+\tilde{S})^{-}= & 2 i \sum_{n \neq 0} c_{n} \sin (n \sigma)\left(1+i \frac{\left(\widehat{\omega}_{n}-n\right)}{\widehat{m}}\right) S_{n} e^{-i \widehat{\omega}_{n} \tau}, \\
(S-\tilde{S})^{-}= & 2 S_{0}^{-} \cosh (\widehat{m} \sigma)+2 S_{0}^{-} \sinh (\widehat{m} \sigma) \\
& +2 \sum_{n \neq 0} c_{n}\left[\cos (n \sigma) \frac{n}{\widehat{\omega}_{n}}\left(1+i \frac{\left(\widehat{\omega}_{n}-n\right)}{\widehat{m}}\right)\right. \\
& \left.+\sin (n \sigma) \frac{\widehat{m}}{\widehat{\omega}_{n}}\left(1+i \frac{\left(\widehat{\omega}_{n}-n\right)}{\widehat{m}}\right)\right] S_{n}^{-} e^{-i \widehat{\omega}_{n} \tau} .
\end{aligned}
$$


The bosonic fields $x^{i^{\prime}}$ have an expansion of the form (3.5). On the other hand, the mode expansion for the bosonic fields in the first four directions is ${ }^{5}$

$$
\begin{aligned}
& x^{i}=\left(x_{0}^{i}+p_{0}^{i} \tau\right) e^{\widehat{m} \sigma} \\
&+2 i \sum_{l \neq 0} \frac{l}{\widehat{\omega}_{l}(l-i \widehat{m})} \alpha_{l}^{i} e^{-i \widehat{\omega}_{l} \tau} \cos (l \sigma)+2 i \sum_{l \neq 0} \frac{\widehat{m}}{\widehat{\omega}_{l}(l-i \widehat{m})} \alpha_{l}^{i} e^{-i \widehat{\omega}_{l} \tau} \sin (l \sigma), \\
& \mathcal{P}^{i}=p_{0}^{i} e^{\widehat{m} \sigma}+2 \sum_{l \neq 0} \frac{l}{(l-i \widehat{m})} \alpha_{l}^{i} e^{-i \widehat{\omega}_{l} \tau} \cos (l \sigma)+2 \sum_{l \neq 0} \frac{\widehat{m}}{(l-i \widehat{m})} \alpha_{l}^{i} e^{-i \widehat{\omega}_{l} \tau} \sin (l \sigma), \quad \\
& x^{i}=\widehat{m}\left(x_{0}^{i}+p_{0}^{i} \tau\right) e^{\widehat{m} \sigma} \\
& \quad-2 i \sum_{l \neq 0} \frac{l^{2}}{\widehat{\omega}_{l}(l-i \widehat{m})} \alpha_{l}^{i} e^{-i \widehat{\omega}_{l} \tau} \sin (l \sigma)+2 i \sum_{l \neq 0} \frac{\widehat{m} l}{\widehat{\omega}_{l}(l-i \widehat{m})} \alpha_{l}^{i} e^{-i \widehat{\omega}_{l} \tau} \cos (l \sigma) .
\end{aligned}
$$

Using these expansions it is straightforward to show that the positive $S O(4)$ chirality component of the difference of the two supercharges (see (3.11))

$$
\mathcal{Q}^{+} \equiv \frac{1}{2}(1+\Pi) \mathcal{Q}=\frac{1}{2 \sqrt{X^{+}}} \int_{0}^{\pi} d \sigma\left[\mathcal{P}^{I} \gamma^{I}(\tilde{S}-S)+x^{\prime I} \gamma^{I}(S+\tilde{S})+\widehat{m} x^{I} \gamma^{I} \Pi(S+\tilde{S})\right]^{+}
$$

and the negative $S O(4)$ chirality component of the sum of the two supercharges

$$
\overline{\mathcal{Q}}^{-} \equiv \frac{1}{2}(1+\Pi) \overline{\mathcal{Q}}=\frac{1}{2 \sqrt{X^{+}}} \int_{0}^{\pi} d \sigma\left[\mathcal{P}^{I} \gamma^{I}(S+\tilde{S})-x^{\prime I} \gamma^{I}(S-\tilde{S})+\widehat{m} x^{I} \gamma^{I} \Pi(S-\tilde{S})\right]^{-}
$$

are conserved. Therefore, this system preserves eight of the dynamical supersymmetries as was the case with the $(0,0)-(0,0)$ system. Furthermore, none of the kinematical supersymmetries is conserved. This follows from the fact that the integrals of the equations in (4.16) obtain contributions from the non-zero modes.

\section{Relation to $(2,2)$ world-sheet supersymmetry}

In earlier sections we have have constructed class II $D$-branes that preserve half of the light-cone gauge dynamical supersymmetries for the cases $(0,0),(4,0)$ and $(0,4)$. From the point of view of our construction it seems that none of the other class II $D$-branes (i.e. the cases $(1,1),(2,2),(3,3),(4,4))$ possess eight unbroken dynamical supersymmetries. In a separate approach the $D$-branes that preserve some supersymmetry in a generic $p p$-wave background with $(2,2)$ world-sheet supersymmetry [5] were recently analysed in [8]. Apart from some oblique branes (see below), the only supersymmetric branes that were found in [8] were the cases $(0,0),(4,0)$ and $(0,4)$. We would like to explain how our results fit in with those of 88 .

The generalised $p p$-wave backgrounds of [5] can be expressed in terms of string theories with $(2,2)$ world-sheet supersymmetry. These backgrounds preserve at least four of the sixteen dynamical light-cone gauge supersymmetries. More precisely, the four parameters

\footnotetext{
${ }^{5}$ We thank Y. Michishita for correcting an error in an earlier version of this paper. This mode expansion was described before, in a different context, in [15].
} 
of the $(2,2)$ world-sheet supercharges are interpreted as four components of the space-time Killing spinor identified in [5] and are parameterized by two complex constants, $\alpha$ and $\zeta$. In the plane-wave background of interest to us, this is only a sub-symmetry of the complete fermionic symmetry of the background. In fact, the four components of the dynamical Killing spinor transform in a certain spinor representation of the diagonal $S O(4)$ subgroup of $S O(4) \times S O(4)$.

This can be seen from the expression for the spinor as given in equation (A.5) of [5]. The piece of the Killing spinor proportional to $\alpha$ is identified with the spinor state $\psi=(+1 / 2,+1 / 2,+1 / 2,+1 / 2)$ which is the bottom state of the $\mathbf{8}_{\mathbf{s}}$ spinor representation of $S O(8)$. The piece proportional to $\zeta$ is $\chi=(-1 / 2,-1 / 2,-1 / 2,-1 / 2)$, which is the top state of $\mathbf{8}_{\mathbf{s}}$.

Decomposing $\mathbf{8}_{\mathbf{s}}$ with respect to the standard embedding of $S O(4) \times S O(4)$ in $S O(8)$ gives

$$
\mathbf{8}_{\mathbf{s}}=\left(\mathbf{2}_{+} \otimes \mathbf{2}_{+}\right) \oplus\left(\mathbf{2}_{-} \otimes \mathbf{2}_{-}\right),
$$

where $\mathbf{2}_{ \pm}$denote the complex two-dimensional spinors of $S O(4)$ with chiralities \pm . The states $\psi$ and $\chi$ lie in the first product together with the states $(+1 / 2,+1 / 2,-1 / 2,-1 / 2)$ and $(-1 / 2,-1 / 2,+1 / 2,+1 / 2)$. Decomposing $\mathbf{2}_{+} \otimes \mathbf{2}_{+}$with respect to the diagonal $S O(4)$, gives

$$
\mathbf{2}_{+} \otimes \mathbf{2}_{+}=\mathbf{2}_{+} \oplus \mathbf{1} \oplus \mathbf{1}
$$

where $\psi$ and $\chi$ generate precisely the $\mathbf{2}_{+}$of the diagonal $S O(4)$, while the other two states are singlets. Similarly, $\mathbf{2}_{-} \otimes \mathbf{2}_{-}$can be decomposed with respect to the diagonal $S O(4)$, and it is easy to see that

$$
\mathbf{2}_{-} \otimes \mathbf{2}_{-}=\mathbf{2}_{-} \oplus \mathbf{1} \oplus \mathbf{1}
$$

Thus the four (real) supercharges considered in [5] transform in the $\mathbf{2}_{+}$representation of the diagonal $S O(4)$, and are in fact uniquely characterised by this property.

Next we want to analyse which of these supersymmetries are preserved in the presence of a $D$-brane ${ }^{6}$. Let us concentrate on the sixteen dynamical supersymmetries of the lightcone gauge type IIB theory. These transform in the $\mathbf{8}_{\mathbf{s}}$ of $S O(8)$ and therefore have chirality + with respect to the $S O(1,1)$ of the light-cone gauge. The dynamical supersymmetries that are preserved by a brane are precisely those that are invariant under the action of $\hat{\Gamma}=\prod_{i \in \mathcal{N}} \gamma^{i}$, where the product is over the gamma-matrices associated with the worldvolume directions of the brane. In light-cone gauge, this translates into the statement that the dynamical supersymmetries in $\mathbf{8}_{\mathbf{s}}$ that survive are those that are invariant under $\Gamma=\prod_{i \in \mathcal{N}^{\prime}} \gamma^{i}$, where $\mathcal{N}^{\prime}$ is the set of transverse world-volume directions. This condition selects out precisely half of the eight complex states.

In relating the results of [8] to our case (where the background preserves the maximal amount of supersymmetry) one has to bear in mind two further restrictions made in [8]:

(i) Since a generic background with $(2,2)$ world-sheet supersymmetry only possesses the space-time Killing spinors described above, all the branes found in 8] preserve

\footnotetext{
${ }^{6}$ We are here discussing the lorentzian $(+,-; r, s)$-branes in order to compare with 8$]$.
} 
a linear combination of the spinors $\psi$ and $\chi$. On the other hand, the maximally supersymmetric background that is considered here, may (and does) possess $D$-branes that preserve half the dynamical supersymmetries, none of which lie in the subspace spanned by $\psi$ and $\chi$. Since these do not preserve the $(2,2)$ supersymmetry of [8] these branes are absent from the analysis of [8].

(ii) In the analysis of [8] an ansatz is made for $\epsilon_{-}$that is only the most general ansatz if the $D$-brane does not preserve any kinematical supersymmetries (which correspond to solutions of the homogeneous $\epsilon_{-}$equation of [5]).

Since all the class I branes preserve half the kinematical supersymmetries, point (ii) above implies that they should not appear in the analysis of [8], and this is indeed the case. Actually, all class I branes, except for certain $(1,3)$ and $(3,1)$ branes, only preserve dynamical supersymmetries that lie outside the subspace spanned by $\psi$ and $\chi$, and therefore do not appear to be supersymmetric from the analysis of [8] because of (i).

As regards the class II branes, suppose that $\Gamma$ is a product of $\gamma_{i}$ matrices (as is the case for the $(+,-; r, s)$ branes). Then for each $(r, s)$ in class II, there exists a configuration for which $\Gamma$ leaves the space spanned by $\psi$ and $\chi$ invariant. In order to see this one can use the representation (up to a suitable normalisation)

$$
\gamma^{i}=\left(b^{i}+b^{+i}\right), \quad i=1,2,3,4, \quad \gamma^{4+i}=i\left(b^{i}-b^{+i}\right), \quad i=1,2,3,4,
$$

where $b^{i}$ and $b^{+i}, i=1,2,3,4$, act as step operators on the four entries of the spinor states $( \pm 1 / 2, \pm 1 / 2, \pm 1 / 2, \pm 1 / 2)$. Furthermore, the supersymmetric class II branes we have found - namely the $(0,0),(4,0)$ and $(0,4)$ branes - do not preserve any kinematical supersymmetries, and thus the analysis of [8] is applicable for them. Our results for these branes are therefore in agreement with the findings of 87 .

Finally, the oblique branes that were found in [8] preserve a two complex dimensional subspace of the space spanned by $\psi$ and $\chi$, but probably not half of the supersymmetries that are present in our cases. Within the context of our analysis it is therefore not surprising that we have not encountered them. It should be straightforward to generalise our construction in order to describe them as well.

We can also use the connection with $(2,2)$ world-sheet supersymmetry to confirm the form of the modified Neumann boundary conditions (4.14). The coupling of the $(+,-; 4,0)$ brane to the background five-form field induces non-zero Born-Infeld flux $F_{+i}$ that is determined in terms of the superpotential $W$ (see equation (4.14) of [8]). For the maximally supersymmetric plane-wave background the superpotential $W$ is quadratic in the transverse coordinates, and $F_{+i}$ is therefore proportional to $\mu x^{i}$. The open string boundary condition for a brane in the presence of this Born-Infeld flux is then

$$
\left(\partial_{\sigma} x^{i}+F_{+i} \partial_{\tau} x^{+}\right)=0
$$

In the usual open string light-cone gauge that is appropriate for time-like branes $x^{+}=p^{+} \tau$, and thus (5.5) becomes

$$
x^{i}-m x^{i}=0
$$


where $m=\mu p^{+}$. Taking into account that the analysis of section is formulated for euclidean branes, and that the mass-parameter in the corresponding open string light-cone gauge is $\widehat{m}$ rather than $m$, (5.6) agrees precisely with the string equation we found in (4.14).

The condition (5.5) agrees also with equation (8.12) of [7]. There it was argued that the derivative of the condition follows from the dynamics of the $(+,-; 4,0)$-brane described by the sum of the Dirac-Born-Infeld lagrangian and the Wess-Zumino term. The latter is proportional to $\int d A \wedge F_{5}$, where $A$ is the Born-Infeld vector potential, $F_{5}$ the background five-form field strength, and the integral is over a seven dimensional surface that bounds the brane.

\section{The classical $D$-instanton}

In this section we will describe the classical supergravity $D$-instanton solution that corresponds to the boundary state we constructed in section 2. We begin by reviewing the description of the IIB $D$-instanton in flat space.

\subsection{Review of the flat space description}

The euclidean field equations of classical Type IIB supergravity theory possess a $D$ instanton solution in which the dilaton and the Ramond-Ramond $(R R)$ scalar fields have nontrivial profiles in the Einstein frame, while all other fields are trivial [16]. The BPS condition relates the $R R$ scalar $\left(C^{(0)}\right)$ to the dilaton $\phi$. The quantity $e^{\phi}$ satisfies the ten-dimensional equation for a scalar Green function,

$$
\nabla_{x}^{2} e^{\phi}=2 \pi|K| \delta^{(10)}\left(x-x_{0}\right)
$$

where $x_{0}$ is the position of the instanton. The $R R$ scalar is given by $d \hat{C}^{(0)}=d e^{-\phi}$, where $d \hat{C}^{(0)}=i d C^{(0)}$ (the factor of $i$ arising due to the effects of the Wick rotation to euclidean signature). The flat space-time solution of this equation is $[16]^{7}$

$$
e^{\phi^{(10)}}=g+h\left(\left|x-x_{0}\right|\right)
$$

where $g=e^{-\phi_{0}}$ is the string coupling constant and

$$
h=\frac{3|K|}{\pi^{4}\left|x-x_{0}\right|^{8}} .
$$

The function $h$ is simply the euclidean scalar field propagator. The corresponding $R R$ scalar field is then given by

$$
C^{(0)}=\chi+i \frac{1}{g+h\left(\left|x-x_{0}\right|\right)}
$$

where $\chi$ is the constant value of the field.

\footnotetext{
${ }^{7}$ This corrects a small numerical mistake in 16 .
} 
The instanton carries a charge $K$ which represents the violation of the Noether symmetry associated with the translation symmetry of the $R R$ scalar, $C^{(0)} \rightarrow C^{(0)}+b$. The Noether current is given by

$$
j_{\mu}=i e^{2 \phi} \partial_{\mu} C^{(0)}
$$

and the charge carried by the $D$-instanton is given by the integral of the radial component of the current over a nine-sphere enclosing the point $x=x_{0}$,

$$
q=\oint d \Sigma_{\mu} i e^{2 \phi} \partial^{\mu} C^{(0)}
$$

where $d \Sigma_{\mu}$ is the element of area on the nine-sphere. A generalization of Dirac's argument for the quantization of magnetic charge in the presence of an electric charge leads to a quantization of $K$ in the presence of a $(4,4)$-brane.

It is straightforward to see that the above solution carries a charge that is an integer multiple of $2 \pi$,

$$
q=\oint d \Sigma_{\mu} \frac{24 K\left(x^{\mu}-x_{0}^{\mu}\right)}{\pi^{4}\left|x-x_{0}\right|^{10}}=2 \pi K .
$$

The $D$-instanton action is equal to $2 \pi|K|$.

The euclidean solution has an interpretation in lorentzian signature space-time as a tunneling process in which the $R R$ charge changes by $K$ units between the initial time $x^{0} \rightarrow-\infty$ and the final time $x^{0} \rightarrow+\infty$. In order to see this, the solution must be continued to lorentzian signature with a suitable $i \epsilon$ prescription which reproduces the causal properties of the Feynman propagator for the non-constant term in (6.2). For our purposes it is of interest to express the fields in light-cone coordinates. In that case the time coordinate is $x^{+}$and the Noether charge of the $R R$ scalar is $i \partial_{+} C^{(0)}$. We may write the solution in the form (letting $X=x-x_{0}$ for convenience)

$$
e^{\phi^{(10)}}=g+\frac{|K|}{2 \pi^{4}} \int_{0}^{\infty} d s s^{3} e^{i s\left(-2 X^{+} X^{-}+\mathbf{X}^{2}+i \epsilon\right)} .
$$

It is natural to Fourier transform this solution with respect to $X^{-}$to express it in terms of the mixed $\left(p^{+}, X^{+}, \mathbf{X}\right)$ representation. This gives a factor of $\delta\left(2 s X^{+}-p^{+}\right)$, which only has support when $p^{+} / X^{+}-\geq 0$ and the result is

$$
\widehat{e^{\phi^{(10)}}} \equiv \frac{1}{2 \pi} \int d X^{-} e^{\phi^{(10)}} e^{i p^{+} X^{-}}=g \delta\left(p^{+}\right)+\frac{|K|\left(p^{+}\right)^{3}}{2\left(2 \pi X^{+}\right)^{4}} e^{i p^{+} \mathbf{X}^{2} / 2 X^{+}} .
$$

This becomes a real solution after a conventional Wick rotation of the light-cone time variable, $X^{+} \rightarrow-i X^{+}$. In contrast to our earlier discussion we shall not perform this Wick rotation in this section. This has to be taken into account when comparing with the results of section 2 .

In light-cone coordinates the charge (6.6) is the difference between the final and initial charges, defined at $X^{+}=+\infty$ and $X^{+}=-\infty$, respectively

$$
q=q_{f}-q_{i}
$$


Here $q_{f}$ and $q_{i}$ are the charges defined at $X^{+}=X_{f}^{+}=+\infty$ and $X^{+}=X_{i}^{+}=-\infty$, respectively. The expression for $q_{f}$ is

$$
q_{f}=\left.\int d \Sigma_{+} \frac{K}{2\left(2 \pi X_{f}^{+}\right)^{4}} \int_{0}^{\infty} d s s^{4} e^{i s\left(-X^{-}+\mathbf{X}^{2} / 2 X_{f}^{+}+i \epsilon / 2 X_{f}^{+}\right)}\right|_{X_{f}^{+}=\infty}
$$

where $d \Sigma_{+}=d^{8} X^{i} d X^{-}$. When expressed in terms of the Fourier transformed solution the result is

$$
q_{f}=\lim _{p^{+} \rightarrow 0_{+}} 2 \pi \int d^{8} X^{i} \frac{K}{2}\left(\frac{p^{+}}{2 \pi X_{f}^{+}}\right)^{4} e^{i p^{+} \mathbf{X}^{2} / 2 X_{f}^{+}} .
$$

Performing the $\mathbf{X}$ integrations in $d \Sigma_{+}$gives

$$
q_{f}=\pi K
$$

where a Wick rotation of $X^{+}$has again been assumed, which makes the integral convergent. Similarly we have $q_{i}=-\pi K$.

\subsection{The classical solution for the plane-wave background}

The solution of (6.1) in a non-trivial conformally flat geometry with vanishing scalar curvature and metric $d s^{2}=\Omega^{2} d x^{2}$ can be straightforwardly written in terms of the harmonic function $h$ in the form

$$
e^{\tilde{\phi}}=g+\Omega^{-4}(x) \Omega^{-4}\left(x_{0}\right) h\left(\left|x-x_{0}\right|\right) .
$$

For example, in the case of $A d S_{5} \times S^{5}, \Omega=\rho^{-1} L$, where $\rho$ is the radial coordinate $\left(\rho^{2} \equiv\right.$ $\left.\sum_{i=5}^{9}\left(x^{i}\right)^{2}\right)$ and $L$ is the scale of the $A d S_{5}$ [17]. The plane-wave background of interest to us has the metric

$$
d s^{2}=-2 d x^{+} d x^{-}-(\pi \mu)^{2}\left(d x^{+}\right)^{2} \mathbf{x}^{2}+d \mathbf{x}^{2},
$$

where $\mathbf{x}$ denotes the eight transverse coordinates. This may be expressed in conformally flat coordinates by transforming to Rosen coordinates (denoted by tilde's)

$$
\tilde{x}^{+}=\frac{1}{\pi \mu} \tan \pi \mu x^{+}, \quad \tilde{\mathbf{x}}=\frac{\mathbf{x}}{\cos \pi \mu x^{+}}, \quad \tilde{x}^{-}=x^{-}+\frac{\pi \mu}{2} \tan \pi \mu x^{+} \mathbf{x}^{2} .
$$

In this system of coordinates the metric becomes

$$
d s_{\text {Rosen }}^{2}=\left(1+\left(\tilde{x}^{+}\right)^{2}\right)^{-1}\left(-2 d \tilde{x}^{+} d \tilde{x}^{-}+d \tilde{\mathbf{x}}^{2}\right)
$$

and thus the conformal prefactor is $\left(1+\left(\tilde{x}^{+}\right)^{2}\right)^{-1}=\cos ^{2} \pi \mu x^{+}$. The invariant finite squared length element is therefore

$$
\Phi_{\text {Rosen }}=\cos \pi \mu x^{+} \cos \pi \mu x_{0}^{+}\left(-2 \tilde{X}^{+} \tilde{X}^{-}+\tilde{\mathbf{X}}^{2}\right) .
$$

In these coordinates the initial and final times are $\tilde{x}^{+}= \pm \infty$, which are at the points where $\cos \pi \mu x^{+}=0$. 
It is easy to transform to the global coordinates (6.15) by substituting the $\tilde{x}$ variables in terms of $x$, giving

$$
\Phi=-\frac{2}{\pi \mu} X^{-} \sin \left(\pi \mu X^{+}\right)-2\left(\mathbf{x}^{2}+\mathbf{x}_{0}^{2}\right) \sin ^{2}\left(\frac{\pi \mu X^{+}}{2}\right)+\mathbf{X}^{2} .
$$

The dilaton profile in the background of a $D$-instanton is therefore,

$$
e^{\tilde{\phi}}=g+\frac{3|K|}{\pi^{4}} \frac{1}{\left(\Phi\left(x, x_{0}\right)+i \epsilon\right)^{4}} .
$$

Apart from the constant, $g$, this is the expression given for the scalar Feynman propagator in [18]. Fourier transforming with respect to $X^{-}$now gives

$$
\widehat{e^{\tilde{\phi}}}=g \delta\left(p^{+}\right)+\frac{|K| \mu^{4}\left(p^{+}\right)^{3}}{2\left(2 \sin \left(\pi \mu X^{+}\right)\right)^{4}} e^{i \frac{\pi \mu p^{+}}{2}}\left(\left(\mathbf{x}^{2}+\mathbf{x}_{0}^{2}\right) \cot \left(\pi \mu X^{+}\right)-2 \mathbf{x} \cdot \mathbf{x}_{0} / \sin \left(\pi \mu X^{+}\right)\right) .
$$

The expression for the charge at a positive value of $X^{+}$due to a $D$-instanton at the origin is then

$$
q_{f}=\lim _{p^{+} \rightarrow 0_{+}} 2 \pi \int d^{8} \mathbf{x} \mathbf{i} \frac{K}{2}\left(\frac{\mu p^{+}}{2 \sin \left(\pi \mu X^{+}\right)}\right)^{4} e^{i p^{+} \pi \mu \mathbf{x}^{2} \cot \left(\pi \mu X_{f}^{+}\right) / 2}=\pi K\left(\cos \left(\pi \mu X_{f}^{+}\right)\right)^{-4},
$$

while at negative $X^{+}$it is

$$
q_{i}=\lim _{p^{+} \rightarrow 0_{-}} 2 \pi \int d^{8} \mathbf{x}^{\mathrm{i}} \frac{K}{2}\left(\frac{p^{+} \mu}{2 \sin \left(\pi \mu X^{+}\right)}\right)^{4} e^{i p^{+} \pi \mu \mathbf{x}^{2} \cot \left(\pi \mu X_{i}^{+}\right) / 2}=-\pi K\left(\cos \left(\pi \mu X_{i}^{+}\right)\right)^{-4}
$$

The dependence of the charge $q_{f}-q_{i}$ on $X_{i}^{+}$and $X_{f}^{+}$is a consequence of the fact that the $R R$ scalar is not massless. It would be interesting to understand the properties of the $D$-instanton in more detail, particularly its relation to the Yang-Mills instanton via the duality conjectured in 四.

\subsection{Supergravity approximation of the interaction energy}

The above discussion implies that one can think of the $D$-instanton at $x_{0}$ as a source of the dilaton and the $R R$ scalar, and that the dilaton and $R R$ scalar profile at $x$ is simply described by the scalar propagator evaluated at $x$ and $x_{0}$. Under this assumption one can calculate the interaction energy between the $D$-instanton and any euclidean $D$-brane in field theory. As we want to demonstrate below, this field theory calculation reproduces the contribution of the lowest lying closed string states in the corresponding string cylinder amplitude. The fact that these calculations agree gives support to the identification of the $D$-instanton boundary state with the classical solution above.

Let us consider a $D$-instanton (i.e. a $(0,0)$-brane) at $\mathbf{y}$, together with another euclidean brane at $\mathbf{0}$. In order to get a non-vanishing answer from the string calculation, the brane at $\mathbf{0}$ will be taken to be, in turn, a $\overline{(0,0)}$-brane, a $(2,0)$-brane and a $(4,2)$-brane. Up to numerical constants, the contributions to the corresponding cylinder diagrams from the zero modes in the closed string channel are given for $(0,0)-\overline{(0,0)}$ by (see $(2.19)$ )

$$
h_{0}(\mathbf{y}, \mathbf{0})(4 \pi m)^{4} \frac{q^{2 m}}{\left(1-q^{m}\right)^{4}} \sim h_{0}(\mathbf{y}, \mathbf{0})\left(8 \pi^{2} \mu p^{+}\right)^{4} \sin ^{-4}\left(\pi \mu X^{+}\right),
$$


while for the case of the $(0,0)$-brane at $\mathbf{y}$ and the $(2,0)$-brane at the origin it is (see (2.22))

$$
\begin{aligned}
j_{0}(\mathbf{y}) & \sinh (\pi m)(4 \pi m)^{2} \frac{q^{2 m}}{\left(1-q^{m}\right)^{3}\left(1+q^{m}\right)} \\
& \sim j_{0}(\mathbf{y}) \sinh \left(2 \pi^{2} \mu p^{+}\right)\left(2 \pi \mu p^{+}\right)^{2} \sin ^{-3}\left(\pi \mu X^{+}\right) \cos ^{-1}\left(\pi \mu X^{+}\right),
\end{aligned}
$$

and for the the case of the $(0,0)$-brane at $\mathbf{y}$ and the $(4,2)$-brane at the origin it is (see (2.24)

$$
\begin{aligned}
j_{0}(\mathbf{y}) \quad & \sinh (\pi m)^{-1}(4 \pi m)^{2} \frac{q^{2 m}}{\left(1-q^{m}\right)^{1}\left(1+q^{m}\right)^{3}} \\
& \sim j_{0}(\mathbf{y}) \sinh \left(2 \pi^{2} \mu p^{+}\right)^{-1}\left(2 \pi \mu p^{+}\right)^{2} \sin ^{-1}\left(\pi \mu X^{+}\right) \cos ^{-3}\left(\pi \mu X^{+}\right) .
\end{aligned}
$$

Notice that the contribution of the $q^{2 m}$ term to these expressions, which can be isolated by letting $X^{+} \rightarrow i \infty$, is proportional to the charge $q_{f}(6.22)$ when this is evaluated in the same limit. This is to be expected since the cylinder diagram factorizes into the product of the $R R$ charges of the ground states of the two branes (one at the origin and one at $X^{+}$) in this limit.

We will now show that the low energy limit of the right-hand sides of these expressions coincide with the quantities obtained from the exchange of a massless scalar field in this background. To see this consider the situation in which there is the (0,0)-brane at $x_{1}^{+}$, $x_{1}^{-}$and $\mathbf{x}_{\mathbf{1}}=\mathbf{y}_{\mathbf{1}}$ in the presence of any euclidean $(r, s)$-brane located at $x_{2}^{+}, x_{2}^{-}$and $\mathbf{x}_{\mathbf{2}} t=\mathbf{y}_{\mathbf{2}}$ (where $\mathbf{x}_{\mathbf{2} t}$ is the transverse position). Then the force is proportional to the scalar propagator between the $(0,0)$-brane and the $(r, s)$-brane integrated over the worldvolume coordinates, $\mathbf{x}_{\mathbf{2}}$, of the $(r, s)$-brane. The propagator is simply equal to

$$
G\left(x_{2} ; x_{1}\right)=(\Phi+i \epsilon)^{-4},
$$

where $\Phi$ is given in (6.19). Next, take the Fourier transform of the propagator with respect to $X^{-}=x_{2}^{-}-x_{1}^{-}$, giving, for $X^{+} \equiv x_{2}^{+}-x_{1}^{+}>0$,

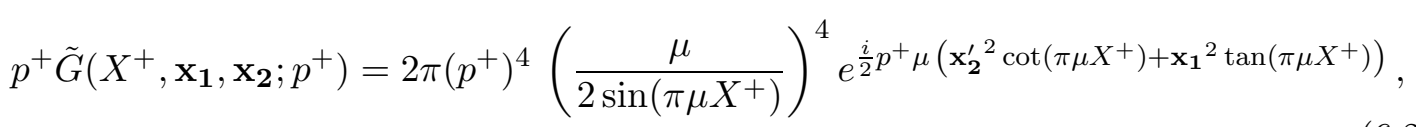

where the extra factor of $p^{+}$has been included to conform to the usual normalisation of the propagator in the light-cone gauge and

$$
\mathbf{x}_{2}^{\prime}=\mathbf{x}_{2}-\frac{\mathbf{x}_{1}}{\cos \pi \mu X^{+}} .
$$

The full interaction is obtained by integrating this expression over the $p+1 \equiv r+s \mathbf{x}_{\mathbf{2}} l$ coordinates tangential to the $(r, s)$-brane (letting $\mathbf{x}_{\mathbf{1}}=\mathbf{y}$ and setting $\mathbf{x}_{\mathbf{2}}=0$ for simplicity),

$$
\begin{aligned}
& p^{+} \tilde{G}\left(X^{+}, \mathbf{y}, \mathbf{x}_{\mathbf{2}}=0 ; p^{+}\right) \\
& =2 \pi \int d^{p+1} \mathbf{x}_{\mathbf{2}}^{\prime}\left(p^{+}\right)^{4}\left(\frac{\mu}{2 \sin \left(\pi \mu X^{+}\right)}\right)^{4} \\
& \quad e^{\frac{i}{2} p^{+} \mu\left(\mathbf{x}_{\mathbf{2}}^{\prime}{ }^{2} \cot \left(\pi \mu X^{+}\right)+\mathbf{y}_{\mathbf{1}}{ }^{2} \cot \left(\pi \mu X^{+}\right)+\mathbf{y}_{\mathbf{t}}{ }^{2} \tan \left(\pi \mu X^{+}\right)\right)} \\
& =2 \pi^{\frac{p+3}{2}}\left(p^{+}\right)^{4-\frac{p+1}{2}}\left(\frac{\mu}{2}\right)^{4-\frac{p+1}{2}}\left(\sin \pi \mu X^{+}\right)^{-4+\frac{p+1}{2}}\left(\cos \pi \mu X^{+}\right)^{-\frac{p+1}{2}} \\
& e^{\frac{i}{2} p^{+} \mu\left(\mathbf{y}_{\mathbf{1}}{ }^{2} \cot \left(\pi \mu X^{+}\right)+\mathbf{y}_{\mathbf{t}}{ }^{2} \tan \left(\pi \mu X^{+}\right)\right) .}
\end{aligned}
$$


This agrees with the expansion of the cylinder expressions 6.24, 6.25) and 6.26) for the cases $p=-1, p=1$ and $p=5$ to leading order in $\alpha^{\prime}$ (remembering that some of the prefactors contain powers of $\left.\sinh \left(2 \pi^{2} \mu p^{+}\right) \simeq 2 \pi^{2} \mu p^{+}\right)$.

\section{Discussion}

In this paper we have shown how to construct various $D$-branes in the type IIB plane wave background that preserve half the dynamical supersymmetries of the background. The instantonic (or euclidean) branes are characterised by the label $(r, s)(r+s=p+$ 1) which defines the number of directions they occupy in the two $S O(4)$ 's. In 11, 9] boundary states for class I $D p$-branes with labels $(0,2),(2,0), 3,1),(1,3),(4,2)$ and $(2,4)$ were constructed and the corresponding cylinder diagrams considered. In those examples the open strings conserved half the kinematical supersymmetries in addition to half the dynamical supersymmetries. The cylinder diagrams joining pairs of these $D$-branes did not vanish (when $p^{+}$is fixed and non-zero). Here we have generalized the earlier discussions to include class II branes with the values $(0,0),(0,4)$ and $(4,0)$. These do not possess the kinematical supersymmetries. On the other hand, the open strings contain massless fermions which cause the cylinder diagrams that link like pairs of branes to vanish. Given our analysis and the independent considerations of [8] it seems that these branes are the only class II branes that preserve dynamical supersymmetries.

The interactions between pairs of $D$-branes associated with cylindrical world-sheets were evaluated for a variety of cases. In each case the cylinder was evaluated in the closed-string channel as an overlap of two boundary states, and also as a trace over the states of an open string joining the two $D$-branes. A rather nontrivial behaviour of these expressions under the $S$ modular transformation was needed in order for the closed-string and open-string calculations to agree.

As already described in [9] our discussion generalises directly to the case of lorentzian signature $D$-branes in which the light-cone coordinates, $x^{ \pm}$, are tangential to the worldvolume and are of the form $(+,-; r, s)$. In fact, the open string analysis we have presented in this paper can be directly applied to time-like branes if we consider the usual open string light cone gauge, and replace $\widehat{m}$ by $m$. Apart from the supersymmetric $D$-branes that had been considered in [12], we have also constructed supersymmetric (class II) branes of types $(+,-; 0,0),(+,-; 4,0)$ and $(+,-; 0,4)$. The latter two carry non-trivial Born-Infeld flux that is induced by the background $R R$ flux. As a consequence, the corresponding 'antibranes' break supersymmetry. As remarked earlier, it should be simple to generalize our discussion to include oblique branes [8] which are oriented in directions that link the first and second $S O(4)$ 's and therefore cannot be represented in the form $(+,-; r, s)$. Furthermore, the light-cone gauges we have used are not appropriate for describing $D$-branes in which $x^{-}$is tangential to the brane with $x^{+}$being transverse (or the converse), which includes the case of 'null branes'.

It would be interesting to understand the properties of these branes from a more

geometrical viewpoint. For example, it is clear that the $(+,-; 0,0)$ D-string arises as a Penrose limit of a non-supersymmetric string wrapping the equator of the five-sphere in 
$A d S^{5} \times S^{5}$ [14, 7]. Likewise, the $(+,-; 0,4)$-brane comes from the baryonic vertex of $A d S^{5} \times S^{5}$, which is a supersymmetric $D 5$-brane wrapping the five-sphere. On the other hand, the $(+,-; 4,0)$-brane originates from a non-supersymmetric $D 5$-brane in $A d S^{5} \times S^{5}$ one of whose axis wraps an equator of the five-sphere while the other axes are aligned with directions in $A d S^{5}$.

The connection of the instantonic branes we have constructed with instantonic branes in $A d S^{5} \times S^{5}$ is more obscure. Understanding this could lead to an understanding of the relation between the $D$-instanton and instanton effects in the dual Yang-Mills field theory. It would also be interesting to understand the effect of the $D$-instanton on the planewave dynamics. Finally, one should be able to analyse the $D$-instanton contributions by considering the effects of the $R^{4}$ and related terms in the effective low energy IIB action 19] in this background.

Note added: while this paper was in the final stages of preparation a preprint by Skenderis and Taylor appeared [21] which contains results that overlap with our section 3.1.1 and appendix C.

\section{Acknowledgements}

We are grateful to Juan Maldacena for useful conversations, and to Pascal Bain, Terry Gannon, Eric Gimon, Leo Pando Zayas, Aninda Sinha and Nemani Suryanarayana for discussions.

MRG is grateful to the Royal Society for a University Research Fellowship. He thanks the Institute for Advanced Study for hospitality while this paper was being completed; his research there was supported by a grant in aid from the Funds for Natural Sciences. We also acknowledge partial support from the PPARC Special Programme Grant 'String Theory and Realistic Field Theory', PPA/G/S/1998/0061 and the EEC contract HPRN2000-00122.

\section{A. Notation and definitions}

We shall be using the same conventions as in [9] (see also [2, 11]). The light-cone lagrangian in the plane-wave background describes eight massive free scalar and eight massive free fermion fields,

$$
\mathcal{L}=\frac{1}{4 \pi}\left(\partial_{+} x^{I} \partial_{-} x^{I}-m^{2}\left(x^{I}\right)^{2}\right)+\frac{i}{2 \pi}\left(S^{a} \partial_{+} S^{a}+\tilde{S}^{a} \partial_{-} \tilde{S}^{a}-2 m S^{a} \Pi_{a b} \tilde{S}^{b}\right),
$$

where $S^{a}$ and $\tilde{S}^{a}$ are $S O(8)$ spinors of the same chirality and $\Pi=\gamma^{1} \gamma^{2} \gamma^{3} \gamma^{4}$. The mass parameter $m$ is defined by $m=2 \pi p^{+} \mu$. The $8 \times 8$ matrices, $\gamma_{a \dot{b}}^{I}$ and $\gamma_{\dot{a} b}^{I}$, are the off-diagonal blocks of the $16 \times 16 S O(8) \gamma$-matrices and couple $S O(8)$ spinors of opposite chirality. The presence of $\Pi$ in the fermionic sector of the lagrangian breaks the symmetry from $S O(8)$ to $S O(4) \times S O(4)$. 
For the bosonic degrees of freedom, the general solution to the equations of motions takes the form

$$
\begin{aligned}
x^{I}(\sigma, \tau) & =\cos (m \tau) x_{0}^{I}+\frac{1}{m} \sin (m \tau) p_{0}^{I}+i \sum_{n \neq 0} \frac{1}{\omega_{n}}\left(e^{-i\left(\omega_{n} \tau-n \sigma\right)} \alpha_{n}^{I}+e^{-i\left(\omega_{n} \tau+n \sigma\right)} \tilde{\alpha}_{n}^{I}\right) \\
\mathcal{P}^{I}(\sigma, \tau) & =\cos (m \tau) p_{0}^{I}-m \sin (m \tau) x_{0}^{I}+\sum_{n \neq 0}\left(e^{-i\left(\omega_{n} \tau-n \sigma\right)} \alpha_{n}^{I}+e^{-i\left(\omega_{n} \tau+n \sigma\right)} \tilde{\alpha}_{n}^{I}\right),
\end{aligned}
$$

where $\mathcal{P}^{I}$ is the canonical momentum associated to $x^{I}, \mathcal{P}^{I}=\dot{x}^{I}$ 迎. The non-zero modes $\alpha_{k}^{I}$ and $\tilde{\alpha}_{k}^{I}$ satisfy the commutation relations

$$
\left[\alpha_{k}^{I}, \alpha_{l}^{J}\right]=\omega_{k} \delta^{I J} \delta_{k,-l}, \quad\left[\alpha_{k}^{I}, \tilde{\alpha}_{l}^{J}\right]=0, \quad\left[\tilde{\alpha}_{k}^{I}, \tilde{\alpha}_{l}^{J}\right]=\omega_{k} \delta^{I J} \delta_{k,-l},
$$

where

$$
\omega_{k}=\operatorname{sign}(k) \sqrt{k^{2}+m^{2}} \quad|k|>0 .
$$

On the other hand, the centre of mass position $x_{0}^{I}$ and the generalised momentum $p_{0}^{I}$ commute with the non-zero modes above, and satisfy

$$
\left[p_{0}^{I}, x_{0}^{J}\right]=-i \delta^{I J}
$$

It is convenient to introduce the creation and annihilation operators

$$
a_{0}^{I}=\frac{1}{\sqrt{2 m}}\left(p_{0}^{I}+i m x_{0}^{I}\right), \quad \bar{a}_{0}^{I}=\frac{1}{\sqrt{2 m}}\left(p_{0}^{I}-i m x_{0}^{I}\right),
$$

in terms of which (A.5) is then simply

$$
\left[\bar{a}_{0}^{I}, a_{0}^{J}\right]=\delta^{I J}
$$

The fermionic fields can be similarly expanded in terms of modes

$$
\begin{aligned}
& S(\sigma, \tau)=S_{0} \cos (m \tau)+\Pi \tilde{S}_{0} \sin (m \tau) \\
& +\sum_{n \neq 0} c_{n}\left[S_{n} e^{-i\left(\omega_{n} \tau-n \sigma\right)}+\frac{i}{m}\left(\omega_{n}-n\right) \Pi \tilde{S}_{n} e^{-i\left(\omega_{n} \tau+n \sigma\right)}\right], \\
& \tilde{S}(\sigma, \tau)=-\Pi S_{0} \sin (m \tau)+\tilde{S}_{0} \cos (m \tau) \\
& +\sum_{n \neq 0} c_{n}\left[\tilde{S}_{n} e^{-i\left(\omega_{n} \tau+n \sigma\right)}-\frac{i}{m}\left(\omega_{n}-n\right) \Pi S_{n} e^{-i\left(\omega_{n} \tau-n \sigma\right)}\right],
\end{aligned}
$$

where $c_{n}$ is defined by

$$
c_{n}=\frac{m}{\sqrt{2 \omega_{n}\left(\omega_{n}-n\right)}} .
$$

The modes $S_{k}^{a}$ and $\tilde{S}_{k}^{a}$, where $a$ is a spinor index of $S O(8)$ and $k \in \mathbb{Z}$, satisfy the anticommutation relations

$$
\left\{S_{k}^{a}, S_{l}^{b}\right\}=\delta^{a b} \delta_{k,-l}, \quad\left\{S_{k}^{a}, \tilde{S}_{l}^{b}\right\}=0, \quad\left\{\tilde{S}_{k}^{a}, \tilde{S}_{l}^{b}\right\}=\delta^{a b} \delta_{k,-l}
$$


It is convenient to introduce the zero-mode combinations,

$$
\theta_{0}^{a}=\frac{1}{\sqrt{2}}\left(S_{0}^{a}+i \tilde{S}_{0}^{a}\right), \quad \bar{\theta}_{0}^{a}=\frac{1}{\sqrt{2}}\left(S_{0}^{a}-i \tilde{S}_{0}^{a}\right),
$$

and further

$$
\begin{aligned}
\theta_{R} & =\frac{1}{2}(1+\Pi) \theta_{0}, & \bar{\theta}_{R} & =\frac{1}{2}(1+\Pi) \bar{\theta}_{0}, \\
\theta_{L} & =\frac{1}{2}(1-\Pi) \theta_{0}, & \bar{\theta}_{L} & =\frac{1}{2}(1-\Pi) \bar{\theta}_{0} .
\end{aligned}
$$

The dynamical supercharges of the closed string theory are given by [2, 11] ${ }^{8}$

$$
\begin{aligned}
& \sqrt{2 p^{+}} Q_{\dot{a}}=\frac{1}{2 \pi} \int_{0}^{2 \pi} d \sigma\left[\mathcal{P}^{I} \gamma^{I} S-x^{\prime I} \gamma^{I} S-m x^{I} \gamma^{I} \Pi \tilde{S}\right] \\
& \sqrt{2 p^{+}} \tilde{Q}_{\dot{a}}=\frac{1}{2 \pi} \int_{0}^{2 \pi} d \sigma\left[\mathcal{P}^{I} \gamma^{I} \tilde{S}+x^{\prime I} \gamma^{I} \tilde{S}+m x^{I} \gamma^{I} \Pi S\right],
\end{aligned}
$$

which in terms of modes is

$$
\begin{aligned}
\sqrt{2 p^{+}} Q_{\dot{a}} & =p_{0}^{I} \gamma_{\dot{a} b}^{I} S_{0}^{b}-m x_{0}^{I}\left(\gamma^{I} \Pi\right)_{\dot{a} b} \tilde{S}_{0}^{b} \\
& +\sum_{n=1}^{\infty}\left(c_{n} \gamma_{\dot{a} b}^{I}\left(\alpha_{-n}^{I} S_{n}^{b}+\alpha_{n}^{I} S_{-n}^{b}\right)+\frac{\mathrm{i} m}{2 \omega_{n} c_{n}}\left(\gamma^{I} \Pi\right)_{\dot{a} b}\left(\tilde{\alpha}_{-n}^{I} \tilde{S}_{n}^{b}-\tilde{\alpha}_{n}^{I} \tilde{S}_{-n}^{b}\right)\right) \\
\sqrt{2 p^{+}} \tilde{Q}_{\dot{a}} & =p_{0}^{I} \gamma_{\dot{a} b}^{I} \tilde{S}_{0}^{b}+m x_{0}^{I}\left(\gamma^{I} \Pi\right)_{\dot{a} b} S_{0}^{b} \\
& +\sum_{n=1}^{\infty}\left(\left(c_{n} \gamma_{\dot{a} b}^{I}\left(\tilde{\alpha}_{-n}^{I} \tilde{S}_{n}^{b}+\tilde{\alpha}_{n}^{I} \tilde{S}_{-n}^{b}\right)-\frac{i m}{2 \omega_{n} c_{n}}\left(\gamma^{I} \Pi\right)_{\dot{a} b}\left(\alpha_{-n}^{I} S_{n}^{b}-\alpha_{n}^{I} S_{-n}^{b}\right)\right)\right.
\end{aligned}
$$

In order to describe the anti-commutation relations of the dynamical supercharges it is useful to introduce $Q_{\dot{a}}^{ \pm}=\frac{1}{\sqrt{2}}\left(Q_{\dot{a}} \pm i \tilde{Q}_{\dot{a}}\right)$. (Note that in contradistinction to (4.18) and (4.19), for example, the index \pm here does not indicate the eigenvalue with respect to the action of П.) Then the anti-commutation relations are [2] $\left\{Q_{\dot{a}}^{ \pm}, Q_{\dot{b}}^{ \pm}\right\}=0$, as well as

$$
\left\{Q_{\dot{a}}^{+}, Q_{\dot{b}}^{-}\right\}=2 \delta_{\dot{a} \dot{b}} H+m\left(\gamma^{i j} \Pi\right)_{\dot{a} \dot{b}} J^{i j}+m\left(\gamma^{i^{\prime} j^{\prime}} \Pi\right)_{\dot{a} \dot{b}} J^{i^{\prime} j^{\prime}}
$$

where $J^{i j}$ are the rotation generators (see [2]) while $H$ is the light-cone hamiltonian $H$ for the closed string in the plane-wave background

$$
\begin{aligned}
2 p^{+} H & =m\left(a_{0}^{I} \bar{a}_{0}^{I}+i S_{0}^{a} \Pi_{a b} \tilde{S}_{0}^{b}+4\right)+\sum_{k=1}^{\infty}\left[\alpha_{-k}^{I} \alpha_{k}^{I}+\tilde{\alpha}_{-k}^{I} \tilde{\alpha}_{k}^{I}+\omega_{k}\left(S_{-k}^{a} S_{k}^{a}+\tilde{S}_{-k}^{a} \tilde{S}_{k}^{a}\right)\right] \\
& =m\left(a_{0}^{I} \bar{a}_{0}^{I}+\theta_{L}^{a} \bar{\theta}_{L}^{a}+\bar{\theta}_{R}^{a} \theta_{R}^{a}\right)+\sum_{k=1}^{\infty}\left[\alpha_{-k}^{I} \alpha_{k}^{I}+\tilde{\alpha}_{-k}^{I} \tilde{\alpha}_{k}^{I}+\omega_{k}\left(S_{-k}^{a} S_{k}^{a}+\tilde{S}_{-k}^{a} \tilde{S}_{k}^{a}\right)\right] .
\end{aligned}
$$

\footnotetext{
${ }^{8}$ We are adopting a slightly different normalisation for the non-zero mode contributions.
} 
In the limit $m \equiv 2 \pi p^{+} \mu \rightarrow 0$ this reduces to the usual light-cone gauge hamiltonian in a flat background [20]. The normal ordering has been chosen in (A.18) with the understanding that $\theta_{L}^{a}$ and $\bar{\theta}_{R}^{a}$ are creation operators while $\bar{\theta}_{L}^{a}$ and $\theta_{R}^{a}$ are annihilation operators.

As is familiar from flat space, the space of states is described by a tensor product of the space generated by the bosonic modes and that generated by the fermionic modes. The ground state of the bosonic space, $|0\rangle_{b}$, is annihilated by the modes $\bar{a}_{0}^{I}$ as well as $\alpha_{k}^{I}$ and $\tilde{\alpha}_{k}^{I}$ with $k>0$ and is non-degenerate since each of the 'zero modes' $a_{0}^{I}$ raises the energy by $m$. Likewise, the non-degenerate ground state in the space spanned by the fermionic operators, $|0\rangle_{f}$, is the state annihilated by $\bar{\theta}_{L}^{a}$ and $\theta_{R}^{a}$, while the creation operators $\theta_{L}^{a}$ and $\bar{\theta}_{R}^{a}$ raise the energy by $m$.

Finally, the kinematical supercharges, $Q_{a} \equiv S_{0}^{a}$ and $\tilde{Q}_{a} \equiv \tilde{S}_{0}^{a}$ do not commute with $H$, but rather satisfy

$$
\left[H, Q_{a}\right]=-\frac{i m}{2 p^{+}} \Pi_{a b} \tilde{Q}_{b}, \quad\left[H, \tilde{Q}_{a}\right]=\frac{i m}{2 p^{+}} \Pi_{a b} Q_{b} .
$$

B. Definition of $f_{i}, g_{i}, \hat{g}_{i}, g_{i, \pm}, \hat{g}_{i, \pm}$

The expressions for the cylinder diagrams with boundaries on pairs of class I branes discussed in [9] are defined by

$$
\begin{aligned}
& f_{1}^{(m)}(q)=q^{-\Delta_{m}}\left(1-q^{m}\right)^{\frac{1}{2}} \prod_{n=1}^{\infty}\left(1-q^{\sqrt{m^{2}+n^{2}}}\right) \\
& f_{2}^{(m)}(q)=q^{-\Delta_{m}}\left(1+q^{m}\right)^{\frac{1}{2}} \prod_{n=1}^{\infty}\left(1+q^{\sqrt{m^{2}+n^{2}}}\right) \\
& f_{3}^{(m)}(q)=q^{-\Delta_{m}^{\prime}} \prod_{n=1}^{\infty}\left(1+q^{\sqrt{m^{2}+(n-1 / 2)^{2}}}\right) \\
& f_{4}^{(m)}(q)=q^{-\Delta_{m}^{\prime}} \prod_{n=1}^{\infty}\left(1-q^{\sqrt{m^{2}+(n-1 / 2)^{2}}}\right)
\end{aligned}
$$

where $\Delta_{m}$ and $\Delta_{m}^{\prime}$ are given as

$$
\begin{aligned}
& \Delta_{m}=-\frac{1}{(2 \pi)^{2}} \sum_{p=1}^{\infty} \int_{0}^{\infty} d s e^{-p^{2} s} e^{-\pi^{2} m^{2} / s} \\
& \Delta_{m}^{\prime}=-\frac{1}{(2 \pi)^{2}} \sum_{p=1}^{\infty}(-1)^{p} \int_{0}^{\infty} d s e^{-p^{2} s} e^{-\pi^{2} m^{2} / s} .
\end{aligned}
$$

These functions satisfy the conditions

$$
f_{1}^{(m)}(q)=f_{1}^{(\widehat{m})}(\tilde{q}), \quad f_{2}^{(m)}(q)=f_{4}^{(\widehat{m})}(\tilde{q}), \quad f_{3}^{(m)}(q)=f_{3}^{(\widehat{m})}(\tilde{q}) .
$$

In this paper various other cylinder diagrams arise that are expressed in terms of generalizations of the above functions. The complete list of these functions is as follows,

$$
g_{1}^{(m)}(t)=4 \pi i m q^{-2 \Delta_{m}} q^{m / 2} \prod_{n=1}^{\infty}\left(1-\left(\frac{\omega_{n}+m}{\omega_{n}-m}\right) q^{\omega_{n}}\right)\left(1-\left(\frac{\omega_{n}-m}{\omega_{n}+m}\right) q^{\omega_{n}}\right)
$$




$$
\begin{gathered}
g_{2}^{(m)}(t)=4 \pi m q^{-2 \Delta_{m}} q^{m / 2} \prod_{n=1}^{\infty}\left(1+\left(\frac{\omega_{n}+m}{\omega_{n}-m}\right) q^{\omega_{n}}\right)\left(1+\left(\frac{\omega_{n}-m}{\omega_{n}+m}\right) q^{\omega_{n}}\right), \\
g_{3}^{(m)}(t)=2 q^{-2 \Delta_{m}^{\prime}} \prod_{n=1}^{\infty}\left(1+\left(\frac{\omega_{n-1 / 2}+m}{\omega_{n-1 / 2}-m}\right) q^{\omega_{n-1 / 2}}\right)\left(1+\left(\frac{\omega_{n-1 / 2}-m}{\omega_{n-1 / 2}+m}\right) q^{\omega_{n-1 / 2}}\right), \\
g_{4}^{(m)}(t)=2 q^{-2 \Delta_{m}^{\prime}} \prod_{n=1}^{\infty}\left(1-\left(\frac{\omega_{n-1 / 2}+m}{\omega_{n-1 / 2}-m}\right) q^{\omega_{n-1 / 2}}\right)\left(1-\left(\frac{\omega_{n-1 / 2}-m}{\omega_{n-1 / 2}+m}\right) q^{\omega_{n-1 / 2}}\right), \\
\hat{g}_{1}^{(\widehat{m})}(\tilde{t})=\tilde{q}^{-\widetilde{\Delta}_{\widehat{m}}} \prod_{l \in \mathcal{M}_{+}}\left(1-\tilde{q}^{\widehat{\omega}_{l}}\right)^{\frac{1}{2}} \prod_{l \in \mathcal{M}_{-}}\left(1-\tilde{q}^{\widehat{\omega}_{l}}\right)^{\frac{1}{2}} \\
\hat{g}_{2}^{(\widehat{m})}(\tilde{t})=\tilde{q}^{-\widetilde{\Delta}_{\hat{m}}} \prod_{l \in \mathcal{M}_{+}}\left(1+\tilde{q}^{\widehat{\omega}_{l}}\right)^{\frac{1}{2}} \prod_{l \in \mathcal{M}_{-}}\left(1+\tilde{q}^{\widehat{\omega}_{l}}\right)^{\frac{1}{2}} \\
\hat{g}_{3}^{(\widehat{m})}(\tilde{t})=\tilde{q}^{-\widehat{\Delta}_{\widehat{m}}} \prod_{l \in \mathcal{P}_{+}}\left(1+\tilde{q}^{\widehat{\omega}_{l}}\right)^{\frac{1}{2}} \prod_{l \in \mathcal{P}_{-}}\left(1+\tilde{q}^{\widehat{\omega}_{l}}\right)^{\frac{1}{2}} \\
\hat{g}_{4}^{(\hat{m})}(\tilde{t})=\tilde{q}^{-\widehat{\Delta}_{\hat{m}}} \prod_{l \in \mathcal{P}_{+}}\left(1-\tilde{q}^{\widehat{\omega}_{l}}\right)^{\frac{1}{2}} \prod_{l \in \mathcal{P}_{-}}\left(1-\tilde{q}^{\widehat{\omega}_{l}}\right)^{\frac{1}{2}} .
\end{gathered}
$$

In these expressions $\widehat{\omega}_{k}=\operatorname{sign}(k) \sqrt{k^{2}+\widehat{m}^{2}}$ and

$$
\begin{aligned}
& \widetilde{\Delta}_{\widehat{m}}=-\frac{1}{(2 \pi)^{2}} \sum_{p=1}^{\infty} \sum_{r=0}^{\infty} c_{r}^{p} \widehat{m} \frac{\partial^{r}}{\left(\partial \widehat{m}^{2}\right)^{r}} \frac{1}{\widehat{m}} \int_{0}^{\infty} d s\left(\frac{-s}{\pi^{2}}\right)^{r} e^{-p^{2} s-\pi^{2} \widehat{m}^{2} / s}, \\
& \widehat{\Delta}_{\widehat{m}}=-\frac{1}{(2 \pi)^{2}} \sum_{p=1}^{\infty}(-1)^{p} \sum_{r=0}^{\infty} c_{r}^{p} \widehat{m} \frac{\partial^{r}}{\left(\partial \widehat{m}^{2}\right)^{r}} \frac{1}{\widehat{m}} \int_{0}^{\infty} d s\left(\frac{-s}{\pi^{2}}\right)^{r} e^{-p^{2} s-\pi^{2} \widehat{m}^{2} / s},
\end{aligned}
$$

where $c_{r}^{p}$ are the Taylor coefficients of the functions

$$
\left(\frac{x+1}{x-1}\right)^{p}+\left(\frac{x-1}{x+1}\right)^{p}=\sum_{r=0}^{\infty} c_{r}^{p} x^{2 r} .
$$

Furthermore, the sets $\mathcal{P}_{ \pm}$and $\mathcal{M}_{ \pm}$are defined by

$$
\begin{array}{cl}
l \in \mathcal{P}_{+} & \text {if } \quad \frac{l+i \widehat{m}}{l-i \widehat{m}}+e^{2 \pi i l}=0, \\
l \in \mathcal{P}_{-} & \text {if } \quad \frac{l-i \widehat{m}}{l+i \widehat{m}}+e^{2 \pi i l}=0, \\
l \in \mathcal{M}_{+} & \text {if } \quad \frac{l+i \widehat{m}}{l-i \widehat{m}}-e^{2 \pi i l}=0, \\
l \in \mathcal{M}_{-} & \text {if } \quad \frac{l-i \widehat{m}}{l+i \widehat{m}}-e^{2 \pi i l}=0 .
\end{array}
$$

We shall show in detail in appendix D that

$$
g_{2}^{(m)}(t)=\hat{g}_{4}^{(\widehat{m})}(\tilde{t}) .
$$


The contour integral method that we use is easily extended to show the other modular properties,

$$
g_{1}^{(m)}(t)=\hat{g}_{1}^{(\widehat{m})}(\tilde{t}), \quad g_{4}^{(m)}(t)=\hat{g}_{2}^{(\widehat{m})}(\tilde{t}), \quad g_{3}^{(m)}(t)=\hat{g}_{3}^{(\widehat{m})}(\tilde{t}) .
$$

For $m \rightarrow 0$, these relations reduce to the standard modular formulae relations between $f_{1}, f_{2}, f_{3}$ and $f_{4}$. Let us, for example, consider the case of the first relation in (B.22). For $\widehat{m}$ small, there are two solutions with $l \in \mathcal{M}_{+}$close to zero at $l= \pm \sqrt{\widehat{m} / \pi}$, and two more solutions with $l \in \mathcal{M}_{-}$close to zero at $l= \pm i \sqrt{\widehat{m} / \pi}$. Consequently, for small $\widehat{m}, \hat{g}_{1}^{(\widehat{m})}(\tilde{t}) \sim 4 \pi \widehat{m} i \tilde{t}^{2} \eta^{2}(\tilde{t})$, while $g_{1}^{(m)}(t) \sim 4 \pi m i \eta^{2}(t)$, and we recover the standard modular transformation formula of the $\eta$-function.

\section{B.1 The factorized $g$-functions}

The functions $g_{i}^{(m)}(t)$ and $\hat{g}_{i}^{(\widehat{m})}(t)$ are naturally expressed in factorized form

$$
g_{i}^{(m)}(t)=g_{i,-}^{(m)}(t) g_{i,+}^{(m)}(t), \quad \hat{g}_{i}^{(\widehat{m})}(t)=\hat{g}_{i,-}^{(\widehat{m})}(\tilde{t}) \hat{g}_{i,+}^{(\widehat{m})}(\tilde{t}) .
$$

The individual factors are defined in such a manner that they have simple transformation properties under the $S$ modular transformation.

We will omit the derivation of these functions and simply state the explicit relations for the cases that are relevant for us. For the closed string functions define

$$
\begin{aligned}
& g_{1,+}^{(m)}(t)=4 \pi i m e^{\mathcal{D}_{m}} q^{m / 2} q^{-\Delta_{m}} \prod_{n=1}^{\infty}\left(1-\left(\frac{\omega_{n}+m}{\omega_{n}-m}\right) q^{\omega_{n}}\right), \\
& g_{1,-}^{(m)}(t)=e^{-\mathcal{D}_{m}} q^{-\Delta_{m}} \prod_{n=1}^{\infty}\left(1-\left(\frac{\omega_{n}-m}{\omega_{n}+m}\right) q^{\omega_{n}}\right) \\
& g_{2,+}^{(m)}(t)=4 \pi m e^{\mathcal{D}_{m}} q^{m / 2} q^{-\Delta_{m}} \prod_{n=1}^{\infty}\left(1+\left(\frac{\omega_{n}+m}{\omega_{n}-m}\right) q^{\omega_{n}}\right), \\
& g_{2,-}^{(m)}(t)=e^{-\mathcal{D}_{m}} q^{-\Delta_{m}} \prod_{n=1}^{\infty}\left(1+\left(\frac{\omega_{n}-m}{\omega_{n}+m}\right) q^{\omega_{n}}\right)
\end{aligned}
$$

where $\mathcal{D}_{m}$ is defined by

$$
\mathcal{D}_{m}=\frac{1}{2 \sqrt{\pi}} \sum_{\hat{n}=1}^{\infty} \int_{0}^{\infty} \frac{d \tilde{s}}{\tilde{s}^{1 / 2}} e^{-\hat{n}^{2} \tilde{s}} \operatorname{Erf}\left(\pi m / \tilde{s}^{1 / 2}\right),
$$

with $\operatorname{Erf}(x)$ the error function

$$
\operatorname{Erf}(x)=1-\frac{2}{\sqrt{\pi}} \int_{0}^{x} d u e^{-u^{2}} .
$$

Under the $S$-modular transformation, these functions transform as

$$
g_{1, \pm}^{(m)}(t)=\hat{g}_{1, \mp}^{(\widehat{m})}(\tilde{t}), \quad g_{2, \pm}^{(m)}(t)=\hat{g}_{4, \mp}^{(\widehat{m})}(\tilde{t}),
$$


where the functions on the right hand side are defined by

$$
\begin{aligned}
& \hat{g}_{1, \pm}^{(\widehat{m})}(\tilde{t})=\tilde{q}^{-\widetilde{\Delta}_{\hat{m}, \pm}} \prod_{l \in \mathcal{M}_{ \pm}}\left(1-\tilde{q}^{\left|\widehat{\omega}_{l}\right|}\right)^{\frac{1}{2}}, \\
& \hat{g}_{4, \pm}^{(\widehat{m})}(\tilde{t})=\tilde{q}^{-\widehat{\Delta}_{\hat{m}, \pm}} \prod_{l \in \mathcal{P}_{ \pm}}\left(1-\tilde{q}^{\left|\widehat{\omega}_{l}\right|}\right)^{\frac{1}{2}} .
\end{aligned}
$$

Here

$$
\begin{aligned}
& \widetilde{\Delta}_{\widehat{m}, \epsilon}=-\frac{1}{(2 \pi)^{2}} \sum_{p=1}^{\infty} \frac{1}{p} \sum_{r=0}^{\infty} d_{r}^{p}\left(\frac{\epsilon}{2 \pi \widehat{m}}\right)^{r} \frac{\partial^{r}}{(\partial p)^{r}} p \int_{0}^{\infty} d s e^{-p^{2} s} e^{-\pi^{2} \widehat{m}^{2} / s}, \\
& \widehat{\Delta}_{\widehat{m}, \epsilon}=-\frac{1}{(2 \pi)^{2}} \sum_{p=1}^{\infty} \frac{(-1)^{p}}{p} \sum_{r=0}^{\infty} d_{r}^{p}\left(\frac{\epsilon}{2 \pi \widehat{m}}\right)^{r} \frac{\partial^{r}}{(\partial p)^{r}} p \int_{0}^{\infty} d s e^{-p^{2} s} e^{-\pi^{2} \widehat{m}^{2} / s},
\end{aligned}
$$

where $d_{r}^{p}$ are the Taylor coefficients of the functions

$$
\left(\frac{x+1}{x-1}\right)^{p}=\sum_{r=0}^{\infty} d_{r}^{p} x^{r} .
$$

\section{Supersymmetry of the open string}

In this appendix the anti-commutation relations of the open-string modes will be determined. We will also show that the supercharge (3.11) is indeed time-independent.

\section{C.1 Anti-commutation relations of the modes}

The anti-commutation relations of the modes $S_{n}$ are fixed by the requirement that the fields $S$ and $\tilde{S}$ satisfy the usual equal time anti-commutation relations

$$
\begin{aligned}
& \left\{S^{a}(\sigma, \tau), S^{b}\left(\sigma^{\prime}, \tau\right)\right\}=2 \pi \delta^{a b} \delta\left(\sigma-\sigma^{\prime}\right), \\
& \left\{S^{a}(\sigma, \tau), \tilde{S}^{b}\left(\sigma^{\prime}, \tau\right)\right\}=0, \\
& \left\{\tilde{S}^{a}(\sigma, \tau), \tilde{S}^{b}\left(\sigma^{\prime}, \tau\right)\right\}=2 \pi \delta^{a b} \delta\left(\sigma-\sigma^{\prime}\right),
\end{aligned}
$$

where $0<\sigma, \sigma^{\prime}<\pi$. These are equivalent to

$$
\begin{aligned}
& \left\{S^{a}(\sigma, \tau) \pm \tilde{S}^{a}(\sigma, \tau), S^{b}\left(\sigma^{\prime}, \tau\right) \pm \tilde{S}^{b}\left(\sigma^{\prime}, \tau\right)\right\}=4 \pi \delta^{a b} \delta\left(\sigma-\sigma^{\prime}\right), \\
& \left\{S^{a}(\sigma, \tau)+\tilde{S}^{a}(\sigma, \tau), S^{b}\left(\sigma^{\prime}, \tau\right)-\tilde{S}^{b}\left(\sigma^{\prime}, \tau\right)\right\}=0 .
\end{aligned}
$$

Given the mode expansions (3.10) it is easy to see that the relation (C.3) as well as the relation in (C.2) involving $S-\tilde{S}$ are satisfied provided that

$$
\left\{S_{n}^{a}, S_{m}^{b}\right\}=\delta^{a, b} \delta_{n,-m}, \quad \text { if } n \neq 0 \text { or } m \neq 0 .
$$

Here one uses the standard identity

$$
\sum_{n=-\infty}^{\infty} e^{i n x}=2 \pi \sum_{r \in \mathbb{Z}} \delta(x+2 \pi r),
$$


as well as the fact that for $0<\sigma, \sigma^{\prime}<\pi, \delta\left(\sigma+\sigma^{\prime}+2 \pi r\right)=0$ for all $r \in \mathbb{Z}$.

Given (C.4) as well as the mode expansion of $S+\tilde{S}$ in (3.10) it is straightforward to determine the contribution of the non-zero modes to $\left\{(S+\tilde{S})(\sigma, \tau),(S+\tilde{S})\left(\sigma^{\prime}, \tau\right)\right\}$, which is

$$
4 \pi \delta^{a b} \delta\left(\sigma-\sigma^{\prime}\right)+2 \delta^{a b} \sum_{n \in \mathbb{Z}} \frac{n^{2}-\widehat{m}^{2}}{n^{2}+\widehat{m}^{2}} e^{i n\left(\sigma+\sigma^{\prime}\right)}+2 \Pi^{a b} \sum_{n \in \mathbb{Z}} \frac{-2 n \widehat{m} i}{n^{2}+\widehat{m}^{2}} e^{i n\left(\sigma+\sigma^{\prime}\right)} .
$$

Note that the two infinite sums now run over all integers; this is immaterial for the second sum, but in the first sum the contribution from $n=0$ cancels the $n=0$ contribution that is necessary in order to produce the $\delta$-function of the first term via (C.5).

The two infinite sums can be evaluated by replacing the sum by a contour integral; for the case of the first sum the relevant contour integral is

$$
\sum_{n \in \mathbb{Z}} \frac{n^{2}-\widehat{m}^{2}}{n^{2}+\widehat{m}^{2}} e^{i n\left(\sigma+\sigma^{\prime}\right)}=-\oint_{C} \frac{d \nu}{1-e^{2 \pi i \nu}} \frac{\nu^{2}-\widehat{m}^{2}}{\nu^{2}+\widehat{m}^{2}} e^{i \nu\left(\sigma+\sigma^{\prime}\right)},
$$

where the contour consists of two lines passing infinitesimally above and below the real axis. Since $0<\sigma+\sigma^{\prime}$ the upper contour can be closed at infinity in the upper half plane and picks up the contribution from the pole at $\nu=i \widehat{m}$ to give

$$
I_{1}=-2 \pi \frac{\widehat{m}}{1-e^{-2 \pi \widehat{m}}} e^{-\widehat{m}\left(\sigma+\sigma^{\prime}\right)}
$$

Similarly, since $\sigma+\sigma^{\prime}<2 \pi$, the lower contour can be closed at infinity in the lower half plane, and gives rise to

$$
I_{2}=2 \pi \frac{\widehat{m}}{1-e^{2 \pi \widehat{m}}} e^{\widehat{m}\left(\sigma+\sigma^{\prime}\right)}
$$

from the pole at $-i \widehat{m}$. By an analogous calculation one finds

$$
\sum_{n \in \mathbb{Z}} \frac{-2 n \widehat{m} i}{n^{2}+\widehat{m}^{2}} e^{i n\left(\sigma+\sigma^{\prime}\right)}=2 \pi \frac{\widehat{m}}{1-e^{-2 \pi \hat{m}}} e^{-\widehat{m}\left(\sigma+\sigma^{\prime}\right)}+2 \pi \frac{\widehat{m}}{1-e^{2 \pi \widehat{m}}} e^{\widehat{m}\left(\sigma+\sigma^{\prime}\right)} .
$$

In order to reproduce (C.2), the sum of (C.8), (C.9) and (C.10) must be cancelled by the contribution from the zero modes to this anti-commutator. This is precisely the case provided that the anti-commutator of the zero modes is given as in (3.14).

\section{C.2 Dynamical supercharge}

The fact that the dynamical supercharge is time independent simply follows by substituting (3.10) and (3.5) into (3.11). In order to see this let us first consider the terms involving bosonic or fermionic zero modes. The contribution that is proportional to $y_{1}^{I} \gamma^{I} S_{n}$ with $n \neq 0$ is equal to (up to the irrelevant prefactor of $\widehat{m} / 2 \sqrt{X^{+}}$)

$$
\begin{gathered}
y_{1}^{I} \gamma^{I} \sum_{n \neq 0} e^{-i \widehat{\omega}_{n} \tau}\left[\int_{0}^{\pi} d \sigma \sinh (\widehat{m} \sigma)\left(\cos (n \sigma)+\frac{\widehat{m}}{n} \sin (n \sigma) \Pi\right)\left[\frac{n}{\widehat{\omega}_{n}}+i \frac{n\left(\widehat{\omega}_{n}-n\right)}{\widehat{m} \widehat{\omega}_{n}} \Pi\right] S_{n}\right. \\
\left.\quad+\int_{0}^{\pi} d \sigma \cosh (\widehat{m} \sigma)\left(\cos (n \sigma) \Pi+\frac{\widehat{m}}{n} \sin (n \sigma)\right)\left[\frac{n}{\widehat{\omega}_{n}}+i \frac{n\left(\widehat{\omega}_{n}-n\right)}{\widehat{m} \widehat{\omega}_{n}} \Pi\right] S_{n}\right] .
\end{gathered}
$$


Using the identities

$$
\begin{aligned}
\int_{0}^{\pi} d \sigma \cosh (\widehat{m} \sigma) \cos (n \sigma) & =\frac{\widehat{m}(-1)^{n}}{\widehat{m}^{2}+n^{2}} \sinh (\widehat{m} \pi), \\
\int_{0}^{\pi} d \sigma \sinh (\widehat{m} \sigma) \sin (n \sigma) & =-\frac{n(-1)^{n}}{\widehat{m}^{2}+n^{2}} \sinh (\widehat{m} \pi), \\
\int_{0}^{\pi} d \sigma \cosh (\widehat{m} \sigma) \sin (n \sigma) & =-\frac{n}{\widehat{m}^{2}+n^{2}}\left[(-1)^{n} \cosh (\widehat{m} \pi)-1\right], \\
\int_{0}^{\pi} d \sigma \sinh (\widehat{m} \sigma) \cos (n \sigma) & =\frac{\widehat{m}}{\widehat{m}^{2}+n^{2}}\left[(-1)^{n} \cosh (\widehat{m} \pi)-1\right]
\end{aligned}
$$

it is easy to see that (C.11) vanishes. The analysis is identical for the term proportional to $\left(y_{2}^{I}-\cosh (\widehat{m} \pi) y_{1}^{I}\right) \gamma^{I} S_{n}$ with $n \neq 0$.

The analysis is similar for the terms proportional to $\alpha_{l}^{I} \gamma^{I} S_{0}$ with $l \neq 0$, i.e.

$$
\begin{aligned}
\frac{2}{\sqrt{X^{+}}} \sum_{l \neq 0} e^{-i \widehat{\omega}_{l} \tau} & {\left[\frac{l}{\widehat{\omega}_{l}} \alpha_{l}^{I} \gamma^{I}\left(\cos (l \sigma) S_{0} \cosh (\widehat{m} \sigma)+\cos (l \sigma) \Pi S_{0} \sinh (\widehat{m} \sigma)\right)\right.} \\
& \left.+\frac{\widehat{m}}{\widehat{\omega}_{l}} \alpha^{I} \gamma^{I}\left(\sin (l \sigma) \Pi S_{0} \cosh (\widehat{m} \sigma)+\sin (l \sigma) S_{0} \sinh (\widehat{m} \sigma)\right)\right]=0
\end{aligned}
$$

Thus, the only terms involving any zero modes are

$$
\begin{aligned}
2 & \frac{\widehat{m}}{\sqrt{X^{+}}} \int_{0}^{\pi} d \sigma\left[\left(y_{1}^{I} \sinh (\widehat{m} \sigma)+\frac{y_{2}^{I}-y_{1}^{I} \cosh (\widehat{m} \pi)}{\sinh (\widehat{m} \pi)} \cosh (\widehat{m} \sigma)\right)\right. \\
& \left.+\left(y_{1}^{I} \cosh (\widehat{m} \sigma)+\frac{y_{2}^{I}-y_{1}^{I} \cosh (\widehat{m} \pi)}{\sinh (\widehat{m} \pi)} \sinh (\widehat{m} \sigma)\right) \gamma^{I}\left(\Pi S_{0}^{\prime} \cosh (\widehat{m} \sigma)+S_{0}^{\prime} \sinh (\widehat{m} \sigma)\right)\right] .
\end{aligned}
$$

Performing the $\sigma$-integrals gives

$$
\mathcal{Q}^{0}=\frac{2}{\sqrt{X^{+}}}\left[y_{2}^{I} \gamma^{I}\left(\cosh (\pi \widehat{m}) S_{0}+\sinh (\pi \widehat{m}) \Pi S_{0}\right)-y_{1}^{I} \gamma^{I} S_{0}\right] .
$$

The non-zero mode contribution proportional to $c_{n} \alpha_{l}^{I} \gamma^{I} S_{n} e^{-i\left(\widehat{\omega}_{l}+\widehat{\omega}_{n}\right) \tau}$ with $n \neq \pm l$ arises with coefficient

$$
\begin{aligned}
\frac{1}{2 \sqrt{X^{+}}} \int_{0}^{\pi} d \sigma & \left\{\cos (l \sigma) \sin (n \sigma) \frac{4 l}{\widehat{\omega}_{l}}\left(\frac{\widehat{m}}{\widehat{\omega}_{n}} \Pi+i \frac{\left(\widehat{\omega}_{n}-n\right)}{\widehat{\omega}_{n}} \mathbb{1}\right)\right. \\
& \left.+\sin (l \sigma) \cos (n \sigma) \frac{4 \widehat{m}}{\widehat{\omega}_{l}}\left(\frac{n}{\widehat{\omega}_{n}} \Pi+i \frac{n\left(\widehat{\omega}_{n}-n\right)}{\widehat{\omega}_{n} \widehat{m}} \mathbb{1}\right)\right\} .
\end{aligned}
$$

Using the identities

$$
\begin{aligned}
& \int_{0}^{\pi} \cos (l \sigma) \sin (n \sigma)=-\frac{n}{n^{2}-l^{2}}\left((-1)^{n+l}-1\right) \\
& \int_{0}^{\pi} \sin (l \sigma) \cos (n \sigma)=\frac{l}{n^{2}-l^{2}}\left((-1)^{n+l}-1\right),
\end{aligned}
$$

we see that each term in the sums in (C.15) vanishes. Similarly one can show that the terms with $n=l \neq 0$ vanish, and thus only terms with $n=-l$ contribute. This proves that the supercharge is time independent. It is also easy to determine the terms with $n=-l$ explicitly. The resulting formula for the supercharge is given in (3.12). 


\section{Modular properties of the cylinder diagrams}

In this appendix the relation (3.39) will be derived. In the process of doing so, we shall also find an explicit expression for $\widehat{\Delta}_{\widehat{m}}$.

\section{D.1 Closed-string perspective}

The procedure for establishing the modular properties of $g_{2}^{(m)}$ begins by considering the logarithm of the expression $(\bar{B} .8)$ and performing a Poisson resummation over the integer $n$. In order to do this, it is important to rewrite (B.8) so that the $n=0$ factor has the same form as the $n>0$ factors. For this purpose it is convenient to introduce a parameter $m_{1}$ and use the relation

$$
\lim _{m_{1} \rightarrow m}\left(1-e^{-2 \pi \sqrt{m^{2}-m_{1}^{2}}}\right)\left(1+\left(\frac{m+m_{1}}{m-m_{1}}\right) q^{m}\right)^{\frac{1}{2}}\left(1+\left(\frac{m-m_{1}}{m+m_{1}}\right) q^{m}\right)^{\frac{1}{2}}=4 \pi m q^{m / 2} .
$$

We can then write

$$
\ln g_{2}^{(m)}=\lim _{m_{1} \rightarrow m}\left(\mathcal{B}_{m_{1}}-\sum_{p=1}^{\infty} \frac{1}{p} e^{-2 \pi p \sqrt{m^{2}-m_{1}^{2}}}\right)+2(2 \pi t) \Delta_{m} .
$$

The term $\mathcal{B}_{m_{1}}$ in $(\mathrm{D} .2)$ is given by

$$
\mathcal{B}_{m_{1}}=-\frac{1}{2} \sum_{p=1}^{\infty} \sum_{n=-\infty}^{\infty} \frac{(-1)^{p}}{p} q^{\omega_{n} p}\left[\left(\frac{\omega_{n}+m_{1}}{\omega_{n}-m_{1}}\right)^{p}+\left(\frac{\omega_{n}-m_{1}}{\omega_{n}+m_{1}}\right)^{p}\right]
$$

with $\omega_{n}=+\sqrt{m^{2}+n^{2}}$. Next use the power series expansion in powers of $\omega_{n}^{2} / m_{1}^{2}$,

$$
\left(\frac{\omega_{n}+m_{1}}{\omega_{n}-m_{1}}\right)^{p}+\left(\frac{\omega_{n}-m_{1}}{\omega_{n}+m_{1}}\right)^{p}=\sum_{r=0}^{\infty} c_{r}^{p}\left(\frac{\omega_{n}}{m_{1}}\right)^{2 r}
$$

which converges for sufficiently large $m_{1}$ for any given value of $n$. The coefficients $c_{r}^{p}$, which will not be needed explicitly, can be used for smaller values of $m_{1}$ since the function on the left-hand side is meromorphic. Using the identity $e^{-z}=\int_{0}^{\infty} d s e^{-s-\pi^{2} z^{2} / 4 s} / \sqrt{\pi s},(\mathrm{D} .3$ ) becomes

$$
\begin{aligned}
\mathcal{B}_{m_{1}} & =-\frac{1}{2 \sqrt{\pi}} \sum_{n=-\infty}^{\infty} \sum_{p=1}^{\infty}(-1)^{p} \sum_{r=0}^{\infty} c_{r}^{p}\left(\frac{\omega_{n}}{m_{1}}\right)^{2 r} \int_{0}^{\infty} \frac{d s}{s^{1 / 2}} e^{-p^{2} s-\pi^{2} t^{2} \omega_{n}^{2} / s} \\
& =-\frac{1}{2 \sqrt{\pi}} \sum_{n=-\infty}^{\infty} \sum_{p=1}^{\infty}(-1)^{p} \sum_{r=0}^{\infty} c_{r}^{p} \frac{\partial^{r}}{\left(\partial t^{2}\right)^{r}} \int_{0}^{\infty} \frac{d s}{s^{1 / 2}}\left(\frac{-s}{\pi^{2} m_{1}^{2}}\right)^{r} e^{-p^{2} s-\pi^{2} t^{2}\left(m^{2}+n^{2}\right) / s} .
\end{aligned}
$$

It is now easy to re-express the sum over $n$ by a Poisson resummation which leads to a sum over $\hat{n}$,

$$
\mathcal{B}_{m_{1}}=\mathcal{B}_{m_{1}}^{\prime}-\frac{1}{2 \pi} \sum_{p=1}^{\infty}(-1)^{p} \sum_{r=0}^{\infty} c_{r}^{p} \frac{\partial^{r}}{\left(\partial t^{2}\right)^{r}} \frac{1}{t} \int_{0}^{\infty} d s\left(\frac{-s}{\pi^{2} m_{1}^{2}}\right)^{r} e^{-p^{2} s-\pi^{2} t^{2} m^{2} / s}
$$


where the $\hat{n}>0$ terms are contained in

$$
\mathcal{B}_{m_{1}}^{\prime}=-\frac{1}{\pi} \sum_{\hat{n}=1}^{\infty} \sum_{p=1}^{\infty}(-1)^{p} \sum_{r=0}^{\infty} c_{r}^{p} \frac{\partial^{r}}{\left(\partial t^{2}\right)^{r}} \frac{1}{t} \int_{0}^{\infty} d s\left(\frac{-s}{\pi^{2} m_{1}^{2}}\right)^{r} e^{-p^{2} s-\pi^{2} t^{2} m^{2} / s} e^{-s \hat{n}^{2} / t^{2}} .
$$

The $\hat{n}=0$ term is the second term in (D.6) which can be rewritten as

$$
-\frac{\tilde{t} \widehat{m}}{2 \pi} \sum_{p=1}^{\infty}(-1)^{p} \sum_{r=0}^{\infty} c_{r}^{p}\left(\frac{m}{m_{1}}\right)^{2 r} \frac{\partial^{r}}{\left(\partial \widehat{m}^{2}\right)^{r}} \frac{1}{\widehat{m}} \int_{0}^{\infty} d s\left(\frac{-s}{\pi^{2}}\right)^{r} e^{-p^{2} s-\pi^{2} \widehat{m}^{2} / s} .
$$

In the limit $m_{1} \rightarrow m$ this has the form $2 \pi \tilde{t} \widehat{\Delta}_{\widehat{m}}$, where $\widehat{\Delta}_{\widehat{m}}$ will be identified with the off-set for the open string

$$
\widehat{\Delta}_{\widehat{m}}=-\frac{1}{(2 \pi)^{2}} \sum_{p=1}^{\infty}(-1)^{p} \sum_{r=0}^{\infty} c_{r}^{p} \widehat{m} \frac{\partial^{r}}{\left(\partial \widehat{m}^{2}\right)^{r}} \frac{1}{\widehat{m}} \int_{0}^{\infty} d s\left(\frac{-s}{\pi^{2}}\right)^{r} e^{-p^{2} s-\pi^{2} \widehat{m}^{2} / s} .
$$

We can check that this expression reduces to the correct offset for the flat space theory in the limit $m \rightarrow 0$. In that limit the only terms that survive are those in which $\partial^{r} /\left(\partial \widehat{m}^{2}\right)^{r}$ acts on the $1 / \widehat{m}$ factor, resulting in

$$
\widehat{\Delta}_{0}=\lim _{\widehat{m} \rightarrow 0} \sum_{p=1}^{\infty}-(-1)^{p} \frac{-1}{4 \pi^{2} p^{2}} \sum_{r=0}^{\infty} c_{r}^{p} \Gamma(2 r+1)\left(-\widehat{m}^{2} \pi^{2} p^{2}\right)^{-r},
$$

which can be written as

$$
\begin{aligned}
\widehat{\Delta}_{0} & =\lim _{\widehat{m} \rightarrow 0} \sum_{p=1}^{\infty}(-1)^{p} \frac{-1}{4 \pi^{2} p^{2}} \int_{0}^{\infty} d y e^{-y} y^{2 r} \sum_{r=0}^{\infty} c_{r}^{p}\left(-\widehat{m}^{2} \pi^{2} p^{2}\right)^{-r} \\
& =\lim _{\widehat{m} \rightarrow 0} \sum_{p=1}^{\infty}(-1)^{p} \frac{-1}{4 \pi^{2} p^{2}} \int_{0}^{\infty} d y e^{-y}\left[\left(\frac{y^{2}-\pi^{2} p^{2} \widehat{m}^{2}}{y^{2}+\pi^{2} p^{2} \widehat{m}^{2}}\right)^{p}+\left(\frac{y^{2}+\pi^{2} p^{2} \widehat{m}^{2}}{y^{2}-\pi^{2} p^{2} \widehat{m}^{2}}\right)^{p}\right] \\
& =2 \sum_{p=1}^{\infty}(-1)^{p} \frac{-1}{4 \pi^{2} p^{2}}=\frac{1}{24}
\end{aligned}
$$

where the fact that $\sum_{p=1}^{\infty}(-1)^{p} / p^{2}=-\pi^{2} / 12$ has been used in the last step. This agrees with the standard expression for the contribution from the Casimir energy to the function $f_{4}$ in the flat space case.

The $\hat{n}>0$ terms in (D.6) can be evaluated explicitly to give (defining $\tilde{s}=s / t^{2}$ ),

$$
\begin{aligned}
\mathcal{B}_{m_{1}}^{\prime}=-\frac{1}{\pi} \sum_{\hat{n}=1}^{\infty} \sum_{p=1}^{\infty} \sum_{r=0}^{\infty}(-1)^{p} c_{r}^{p} \int_{0}^{\infty} d \tilde{s} \sum_{l=0}^{\infty} \frac{\left(-p^{2} \tilde{s}\right)^{l}}{l !} \\
\frac{\Gamma\left(l+r+\frac{3}{2}\right)}{\Gamma\left(l+\frac{3}{2}\right)}\left(\frac{-\tilde{s}}{\pi^{2} m_{1}^{2}}\right)^{r} t^{2 l+1} e^{-\hat{n}^{2} \tilde{s}-\pi^{2} m^{2} / \tilde{s}} .
\end{aligned}
$$

From the definition $(\overline{D .2})$ it is clear that both $\mathcal{B}_{m_{1}}$ and the term $\sum_{p=1}^{\infty} e^{-2 \pi p \sqrt{m^{2}-m_{1}^{2}}} / p$ in (D.2) are separately divergent in the limit $m_{1} \rightarrow m$. For this reason it is important to keep $m_{1} \neq m$ in (D.12). We have now reexpressed $\mathcal{B}_{m_{1}}$ in (D.2) in a form that will be compared with the expression obtained from the open string calculation. 


\section{D.2 Open-string perspective}

We now start with the expression $\widehat{g}_{4}^{(m)}$ in (B.14). In this case the logarithm has the form

$$
\ln \widehat{g}_{4}^{(\widehat{m})}=\mathcal{C}+2 \pi \tilde{t} \widehat{\Delta}_{\widehat{m}}
$$

where

$$
\mathcal{C}_{m_{1}}=-\frac{1}{2 \sqrt{\pi}} \sum_{\hat{n}=1}^{\infty} \sum_{\hat{p}} \int_{0}^{\infty} \frac{d \tilde{s}}{\tilde{s}^{1 / 2}} e^{-\hat{n}^{2} \tilde{s}} e^{-\pi^{2} \tilde{t}^{2}\left(\hat{m}^{2}+\hat{p}^{2}\right) / \tilde{s}} .
$$

Here the sum over $\hat{p}$ runs over both $\mathcal{P}_{+}$and $\mathcal{P}_{-}$, including the value $\hat{p}=0$ in both sectors. The second term on the right-hand side of (D.13) should be given by the Casimir energy of

a two-dimensional boson field on an open-string world-sheet of width $\tilde{t}$ with the appropriate boundary conditions. We will postpone the discussion of this term until later when we will see that it coincides with the expression obtained from the closed-string side in the second term in (D.6).

We will start by considering $\mathcal{C}_{m_{1}}$. In order to proceed we need to perform a Poisson resummation over the values of $\hat{p}$. This is achieved by replacing each term in the $\hat{p}$ sum by a contour integral over a complex variable $\rho$, enclosing the relevant pole at the value of $\hat{p}$ that solves the above relations, giving

$$
\begin{aligned}
\mathcal{C}_{m_{1}}=-\frac{1}{2 \pi \sqrt{\pi}} \oint d \rho \sum_{\hat{n}=1}^{\infty} \int_{0}^{\infty} \frac{d \tilde{s}}{\tilde{s}^{1 / 2}} e^{-\hat{n}^{2} \tilde{s}-\pi^{2} \tilde{t}^{2}\left(\widehat{m}^{2}+\rho^{2}\right) / \tilde{s}} \\
\left.\qquad \frac{\left(\pi+\frac{\widehat{m}_{1}}{\rho^{2}+\widehat{m}_{1}^{2}}\right)}{1+\left(\frac{\rho+i \widehat{m}_{1}}{\rho-i \widehat{m}_{1}}\right) e^{-2 \pi i \rho}}+\frac{\left(\pi-\frac{\widehat{m}_{1}}{\rho^{2}+\widehat{m}_{1}^{2}}\right)}{1+\left(\frac{\rho-i \widehat{m}_{1}}{\rho+i \widehat{m}_{1}}\right) e^{-2 \pi i \rho}}\right] .
\end{aligned}
$$

The contour is the sum of small circles enclosing all the poles arising from real zeroes of the denominators in the square brackets on the right-hand side of this equation. The initial contour may now be deformed into the sum of two straight lines. One of these is a straight line, $L_{1}$, displaced by $\epsilon>0$ above the real axis and running from $\rho=\infty+i \epsilon$ to $\rho=-\infty+i \epsilon$. The other part is the line $L_{2}$, displaced by $-\epsilon$ below the real axis and running from $\rho=-\infty-i \epsilon$ to $\rho=\infty-i \epsilon$.

In order to expand the denominators of the terms in the square brackets in (D.15) in convergent series it is important to choose $\epsilon>\widehat{m}_{1}$. This means that the upper and lower contours are shifted into the upper and lower half $\rho$-plane, respectively. It is therefore important to understand the singularity structure of the integrand in the complex $\rho$-plane in (D.15). A careful analysis reveals that the denominators of the terms in square brackets have no zeroes or singularities away from the real $\rho$ axis. However, the numerators of the terms in the square brackets have poles at $\rho= \pm i \widehat{m}_{1}$ and the residues of these poles contribute when the integration contours are displaced. The total contribution from these two poles to $\mathcal{C}_{m_{1}}$ is

$$
\mathcal{C}_{m_{1}}^{\prime \prime}=-\frac{1}{\sqrt{\pi}} \int_{0}^{\infty} \frac{d \tilde{s}}{\tilde{s}^{1 / 2}} e^{-\hat{n}^{2} \tilde{s}-\pi^{2} \tilde{t}^{2}\left(\widehat{m}^{2}-\widehat{m}_{1}^{2}\right) / \tilde{s}}=-\sum_{\hat{n}=1}^{\infty} \frac{1}{\hat{n}} e^{-2 \pi \hat{n} \sqrt{m^{2}-m_{1}^{2}}}
$$


which matches the second term in brackets in (D.2). The total value of $\mathcal{C}_{m_{1}}$ is therefore

$$
\mathcal{C}_{m_{1}}=\mathcal{C}_{m_{1}}^{L_{1}}+\mathcal{C}_{m_{1}}^{L_{2}}+\mathcal{C}_{m_{1}}^{\prime \prime}
$$

where $\mathcal{C}_{m_{1}}^{L_{1}}$ and $\mathcal{C}_{m_{1}}^{L_{2}}$ denote the terms coming from the integration over the upper and lower contours.

Along the lower contour $L_{2}$ we expand the denominators in the square brackets of (D.15) in powers of $\left(\rho+i \widehat{m}_{1} / \rho-i \widehat{m}_{1}\right) e^{-2 \pi i \rho}$, which gives

$$
\begin{aligned}
\mathcal{C}_{m_{1}}^{L_{2}}=-\frac{1}{2 \pi \sqrt{\pi}} \int_{-\infty}^{\infty} d \rho \sum_{\hat{n}=1}^{\infty} \int_{0}^{\infty} \frac{d \tilde{s}}{\tilde{s}^{1 / 2}} \sum_{p=0}^{\infty}(-1)^{p} e^{-\hat{n}^{2} \tilde{s}-\pi^{2} \tilde{t}^{2}\left(\widehat{m}^{2}+\rho^{2}\right) / \tilde{s}} e^{-2 \pi i \rho p} \\
\\
{\left[\left(\pi+\frac{\widehat{m}_{1}}{\rho^{2}+\widehat{m}_{1}^{2}}\right)\left(\frac{\rho+i \widehat{m}_{1}}{\rho-i \widehat{m}_{1}}\right)^{p}+\left(\pi-\frac{\widehat{m}_{1}}{\rho^{2}+\widehat{m}_{1}^{2}}\right)\left(\frac{\rho-i \widehat{m}_{1}}{\rho+i \widehat{m}_{1}}\right)^{p}\right] . }
\end{aligned}
$$

In order to obtain a convergent series expansion along the upper contour $L_{1}$ it is necessary to expand in powers of $\left(\rho-i \widehat{m}_{1} / \rho+i \widehat{m}_{1}\right) e^{2 \pi i \rho}$. After taking into account the reversal of orientation of the integration this gives $\mathcal{C}_{m_{1}}^{L_{1}}$ of the same form as $\mathcal{C}_{m_{1}}^{L_{2}}$, except that now the sum over $p$ does not include the $p=0$ term,

$$
\mathcal{C}_{m_{1}}^{L_{2}}-\mathcal{C}_{m_{1}}^{L_{1}}=-\frac{1}{\sqrt{\pi}} \int_{-\infty}^{\infty} d \rho \sum_{\hat{n}=1}^{\infty} \int_{0}^{\infty} \frac{d \tilde{s}}{\tilde{s}^{1 / 2}} e^{-\hat{n}^{2} \tilde{s}-\pi^{2} \tilde{t}^{2}\left(\hat{m}^{2}+\rho^{2}\right) / \tilde{s}} .
$$

We will separate out the $p=0$ term by writing

$$
\mathcal{C}_{m_{1}} \equiv \mathcal{C}_{m_{1}}^{L_{1}}+\mathcal{C}_{m_{1}}^{L_{2}}+\mathcal{C}_{m_{1}}^{\prime \prime}=\mathcal{C}_{m_{1}}^{\prime}+\mathcal{C}_{m_{1}}^{\prime \prime}+2(2 \pi t) \Delta_{m}
$$

where

$$
\begin{aligned}
2(2 \pi t) \Delta_{m} & =-\frac{1}{\sqrt{\pi}} \int_{-\infty}^{\infty} d \rho \sum_{\hat{n}=1}^{\infty} \int_{0}^{\infty} \frac{d \tilde{s}}{\tilde{s}^{1 / 2}} e^{-\hat{n}^{2} \tilde{s}-\pi^{2} \tilde{t}^{2}\left(\hat{m}^{2}+\rho^{2}\right) / \tilde{s}} \\
& =-\frac{1}{\tilde{t} \pi} \sum_{\hat{n}=1}^{\infty} \int_{0}^{\infty} d \tilde{s} e^{-\hat{n}^{2} \tilde{s}-\pi^{2} \tilde{t}^{2} \hat{m}^{2} / \tilde{s}} \\
& =-2(2 \pi t) \frac{1}{(2 \pi)^{2}} \sum_{\hat{n}=1}^{\infty} \int_{0}^{\infty} d \tilde{s} e^{-\hat{n}^{2} \tilde{s}-\pi^{2} m^{2} / \tilde{s}} .
\end{aligned}
$$

thus producing the correct off-set of the closed string calculation. Next expand the $p \neq 0$ terms in a power series in $\rho / \widehat{m}_{1}$. The terms with coefficient $\pi$ in the first bracket in (D.18) only involve powers of $\rho^{2} / \widehat{m}_{1}^{2}$. For these terms we have the expansion

$$
2 \cos 2 p \phi \equiv\left(\frac{\rho+i \widehat{m}_{1}}{\rho-i \widehat{m}_{1}}\right)^{p}+\left(\frac{\rho-i \widehat{m}_{1}}{\rho+i \widehat{m}_{1}}\right)^{p}=\sum_{r} c_{r}^{p}(-1)^{r}\left(\rho^{2} / \widehat{m}_{1}^{2}\right)^{r}
$$

where the coefficients are the same as those that arose in the closed string calculation. Note that we can also write

$$
2 i \sin 2 p \phi \equiv\left(\frac{\rho+i \widehat{m}_{1}}{\rho-i \widehat{m}_{1}}\right)^{p}-\left(\frac{\rho-i \widehat{m}_{1}}{\rho+i \widehat{m}_{1}}\right)^{p}=-\frac{i}{p} \frac{d \cos (2 p \phi)}{d \phi}=-\frac{i}{p} \frac{d \cos (2 p \phi)}{d \rho}\left(\frac{d \phi}{d \rho}\right)^{-1} .
$$


Using

$$
e^{2 i \phi}=\left(\frac{\rho+i \widehat{m}_{1}}{\rho-i \widehat{m}_{1}}\right)
$$

we see that

$$
\left(\frac{d \phi}{d \rho}\right)=-\frac{\widehat{m}_{1}}{\rho^{2}+\widehat{m}_{1}^{2}}
$$

Substituting these relations into the expression for $\mathcal{C}_{m_{1}}$ gives

$$
\mathcal{C}_{m_{1}}^{\prime}=\mathcal{C}_{m_{1}}^{e}+\mathcal{C}_{m_{1}}^{o}
$$

where

$$
\mathcal{C}_{m_{1}}^{e}=-\frac{1}{\pi \sqrt{\pi}} \int_{-\infty}^{\infty} d \rho \sum_{\hat{n}=1}^{\infty} \int_{0}^{\infty} \frac{d \tilde{s}}{\tilde{s}^{1 / 2}} \sum_{p=1}^{\infty}(-1)^{p} e^{-\hat{n}^{2} \tilde{s}-\pi^{2} \tilde{t}^{2}\left(\widehat{m}^{2}+\rho^{2}\right) / \tilde{s}} e^{-2 \pi i \rho p} 2 \pi \cos (2 p \phi),
$$

and

$$
\mathcal{C}_{m_{1}}^{o}=-\frac{1}{\pi \sqrt{\pi}} \int_{-\infty}^{\infty} d \rho \sum_{\hat{n}=1}^{\infty} \int_{0}^{\infty} \frac{d \tilde{s}}{\tilde{s}^{1 / 2}} \sum_{p=1}^{\infty}(-1)^{p} e^{-\hat{n}^{2} \tilde{s}-\pi^{2} \tilde{t}^{2}\left(\hat{m}^{2}+\rho^{2}\right) / \tilde{s}} e^{-2 \pi i \rho p} \frac{i}{p} \frac{d \cos (2 p \phi)}{d \rho} .
$$

Integrating the last integral by parts gives two terms where the $\partial / \partial \rho$ acts on $e^{-2 \pi i \rho p}$ and on $e^{-\pi^{2} \tilde{t}^{2} \rho^{2} / \tilde{s}}$. Therefore the full expression for $\mathcal{C}_{m_{1}}^{\prime}$ is

$$
\begin{aligned}
\mathcal{C}_{m_{1}}^{\prime} & =\frac{i}{\pi \sqrt{\pi}} \int_{-\infty}^{\infty} d \rho \sum_{\hat{n}=1}^{\infty} \int_{0}^{\infty} \frac{d \tilde{s}}{\tilde{s}^{1 / 2}} \sum_{p=1}^{\infty} \frac{(-1)^{p}}{p} e^{-2 \pi i \rho p} \cos 2 p \phi \frac{d}{d \rho} e^{-\frac{\pi^{2} \tilde{t}^{2}}{\tilde{s}}\left(\rho^{2}+\widehat{m}^{2}\right)-\hat{n}^{2} \tilde{s}}(\mathrm{D} .29) \\
& =-2 i \sqrt{\pi} \int_{-\infty}^{\infty} d \rho \rho \sum_{\hat{n}=1}^{\infty} \int_{0}^{\infty} \frac{d \tilde{s}}{\tilde{s}^{3 / 2}} \sum_{p=1}^{\infty} \frac{(-1)^{p}}{p} \tilde{t}^{2} e^{-2 \pi i \rho p} \cos 2 p \phi e^{-\frac{\pi^{2} \tilde{t}^{2}}{\tilde{s}}\left(\rho^{2}+\hat{m}^{2}\right)-\hat{n}^{2} \tilde{s}}
\end{aligned}
$$

Substituting the expansion for $\cos 2 p \phi$ gives

$$
\begin{aligned}
\mathcal{C}_{m_{1}}^{\prime}=-i \sqrt{\pi} \int_{-\infty}^{\infty} d \rho \sum_{\hat{n}=1}^{\infty} \int_{0}^{\infty} \frac{d \tilde{s}}{\tilde{s}^{3 / 2}} \sum_{p=1}^{\infty} \frac{(-1)^{p}}{p} \widetilde{t}^{2} \\
\sum_{r=0}^{\infty} c_{r}^{p}(-1)^{r}\left(\frac{\rho^{2}}{\widehat{m}_{1}^{2}}\right)^{r} \rho e^{-2 \pi i \rho p} e^{-\frac{\pi^{2} \tilde{t}^{2}}{\tilde{s}}\left(\rho^{2}+\widehat{m}^{2}\right)-\hat{n}^{2} \tilde{s}} .
\end{aligned}
$$

We can replace the odd power of $\rho$ with the factor $i \partial / 2 \pi \partial p$ acting only on the exponential to its right. The remaining powers of $\rho^{2}$ can be replaced by derivatives with respect to $\tilde{t}^{2}$. This gives

$$
\begin{aligned}
\mathcal{C}_{m_{1}}^{\prime}= & \frac{1}{2 \sqrt{\pi}} \int_{-\infty}^{\infty} d \rho \sum_{\hat{n}=1}^{\infty} \int_{0}^{\infty} \frac{d \tilde{s}}{\tilde{s}^{3 / 2}} \sum_{p=1}^{\infty} \frac{(-1)^{p}}{p} \tilde{t}^{2} \\
& \sum_{r=0}^{\infty} c_{r}^{p}\left(\frac{\tilde{s}}{\pi^{2} \widehat{m}_{1}^{2}}\right)^{r} e^{-\frac{\pi^{2} \tilde{t}^{2} \hat{m}^{2}}{\tilde{s}}} \frac{\partial^{r}}{\left(\partial \tilde{t}^{2}\right)^{r}} \frac{\partial}{\partial p} e^{-2 \pi i \rho p} e^{-\frac{\pi^{2} \tilde{t}^{2} \rho^{2}}{\tilde{s}}-\hat{n}^{2} \tilde{s}}
\end{aligned}
$$


We can now do the $\rho$ integral very simply by completing a square, changing variables from $\rho$ to $\rho^{\prime}=\rho-i p \tilde{s} / \pi \tilde{t}^{2}$ and shifting the $\rho^{\prime}$ integration contour to lie along the real axis,

$$
\begin{aligned}
\mathcal{C}_{m_{1}}^{\prime} & =\frac{1}{2 \pi} \sum_{\hat{n}=1}^{\infty} \int_{0}^{\infty} \frac{d \tilde{s}}{\tilde{s}} \sum_{p=1}^{\infty} \frac{(-1)^{p}}{p} \tilde{t}^{2} \sum_{r=0}^{\infty} c_{r}^{p}\left(\frac{\tilde{s}}{\pi^{2} \widehat{m}_{1}^{2}}\right)^{r} e^{-\frac{\pi^{2} \tilde{t}^{2} \hat{m}^{2}}{\tilde{s}}} \frac{\partial^{r}}{\left(\partial \tilde{t}^{2}\right)^{r}} \frac{\partial}{\partial p} \frac{1}{\tilde{t}} e^{-\frac{p^{2} \tilde{s}}{\tilde{t}^{2}}-\hat{n}^{2} \tilde{s}} \\
& =-\frac{1}{\pi} \sum_{\hat{n}=1}^{\infty} \int_{0}^{\infty} d \tilde{s} \sum_{p=1}^{\infty}(-1)^{p} \tilde{t}^{2} \sum_{r=0}^{\infty} c_{r}^{p}\left(\frac{\tilde{s} \tilde{t}^{2}}{\pi^{2} m_{1}^{2}}\right)^{r} e^{-\pi^{2} m^{2} / \tilde{s}} \frac{\partial^{r}}{\left(\partial \tilde{t}^{2}\right)^{r}} \frac{1}{\tilde{t}^{3}} e^{-\frac{p^{2} \tilde{s}}{\tilde{t}^{2}}-\hat{n}^{2} \tilde{s}},(\mathrm{D}
\end{aligned}
$$

where $\widehat{m}_{1}=m_{1} / \tilde{t}$ has been used in the final line. Expanding the exponential involving $\tilde{t}$, and differentiating term by term, gives

$$
\begin{aligned}
\mathcal{C}_{m_{1}}^{\prime}=-\frac{1}{\pi} \sum_{\hat{n}=1}^{\infty} \int_{0}^{\infty} d \tilde{s} \sum_{p=1}^{\infty}(-1)^{p} \sum_{r=0}^{\infty} c_{r}^{p}\left(\frac{\tilde{s}}{\pi^{2} m_{1}^{2}}\right)^{r} e^{-\pi^{2} m^{2} / \tilde{s}-\hat{n}^{2} \tilde{s}} \\
\sum_{l=0}^{\infty} \frac{\left(-p^{2} \tilde{s}\right)^{l}}{l !} \frac{\Gamma(-l-1 / 2)}{\Gamma(-l-r-1 / 2)} \tilde{t}^{-(2 l+1)} \\
=-\frac{1}{\pi} \sum_{\hat{n}=1}^{\infty} \int_{0}^{\infty} d \tilde{s} \sum_{p=1}^{\infty}(-1)^{p} \sum_{r=0}^{\infty} c_{r}^{p}\left(\frac{-\tilde{s}}{\pi^{2} m_{1}^{2}}\right)^{r} e^{-\pi^{2} m^{2} / \tilde{s}-\hat{n}^{2} \tilde{s}} \\
\sum_{l=0}^{\infty} \frac{\left(-p^{2} \tilde{s}\right)^{l}}{l !} \frac{\Gamma(l+r+1 / 2)}{\Gamma(l+3 / 2)} \tilde{t}^{-(2 l+1)} .
\end{aligned}
$$

This reproduces (D.12) upon setting $\tilde{t}=1 / t$.

We now turn to the second term in (D.13), which is proportional to the Casimir energy of a two-dimensional field. This is equal to the difference of the vacuum energy on a finite strip of width $\tilde{t}$ and an infinitely wide strip. Using a standard argument, the vacuum energy on the finite width strip is given by setting $\hat{n}=0$ in (D.14). Each term in the resulting series in the $\hat{p}$ sum is divergent. However, after the Poisson resummation the divergence is entirely in the $p=0$ term, which, in the $m_{1} \rightarrow m$ limit, is the $\tilde{t}$-independent term that has to be subtracted in obtaining the Casimir energy. Therefore, the Casimir energy is obtained by setting $\hat{n}=0$ in the summand of the right-hand side of (D.32), which gives

$$
2 \pi \tilde{t} \widehat{\Delta}=-\frac{1}{\pi} \int_{0}^{\infty} d \tilde{s} \sum_{p=1}^{\infty}(-1)^{p} \tilde{t}^{2} \sum_{r=0}^{\infty} c_{r}^{p}\left(\frac{\tilde{s} \tilde{t}^{2}}{\pi^{2} m_{1}^{2}}\right)^{r} e^{-\pi^{2} m^{2} / \tilde{s}} \frac{\partial^{r}}{\left(\partial \tilde{t}^{2}\right)^{r}} \frac{1}{\tilde{t}^{3}} e^{-\frac{p^{2} \tilde{s}}{\tilde{t}^{2}}}
$$

thus reproducing (D.9). This completes the argument.

\section{References}

[1] R.R. Metsaev, Type IIB Green-Schwarz superstring in plane wave Ramond-Ramond background, Nucl. Phys. B625, 70 (2002); hep-th/0112044.

[2] R.R. Metsaev, A.A. Tseytlin, Exactly solvable model of superstring in plane wave Ramond-Ramond background, Phys. Rev. D65, 126004 (2002); hep-th/0202109. 
[3] M. Blau, J. Figueroa-O'Farrill, C. Hull, G. Papadopoulos, Penrose limits and maximal supersymmetry, Class. Quant. Grav. 19, L87 (2002); hep-th/0201081.

[4] D. Berenstein, J. Maldacena, H. Nastase, Strings in flat space and pp waves from $\mathcal{N}=4$ Super Yang Mills, JHEP 0204, 013 (2002); hep-th/0202021.

[5] J. Maldacena, L. Maoz, Strings on pp-waves and massive two dimensional field theories, hep-th/0207284.

[6] N. Berkovits, J. Maldacena, $N=2$ superconformal description of superstring in Ramond-Ramond plane wave backgrounds, JHEP 0210, 059 (2002); hep-th/0208092.

[7] K. Skenderis, M. Taylor, Branes in AdS and pp-wave spacetimes, JHEP 0206, 025 (2002); hep-th/0204054.

[8] Y. Hikida, S. Yamaguchi, D-branes in pp-waves and massive theories on worldsheet with boundary, hep-th/0210262.

[9] O. Bergman, M.R. Gaberdiel, M.B. Green, D-brane interactions in type IIB plane-wave background, hep-th/0205183.

[10] M.B. Green, M. Gutperle, Light-cone supersymmetry and D-branes, Nucl. Phys. B476, 484 (1996); hep-th/9604091.

[11] M. Billo, I. Pesando, Boundary states for GS superstrings in an Hpp wave background, Phys. Lett. B536, 121 (2002); hep-th/0203028.

[12] A. Dabholkar, S. Parvizi, Dp branes in PP-wave background, hep-th/0203231.

[13] P. Bain, P. Meessen, M. Zamaklar, Supergravity solutions for D-branes in Hpp-wave backgrounds, hep-th/0205106.

[14] P. Bain, K. Peeters, M. Zamaklar, D-branes in a plane wave from covariant open strings, hep-th/0208038.

[15] Y. Michishita, D-branes in NSNS and RR pp-wave backgrounds and S-duality, JHEP 0210, 048 (2002); hep-th/0206131.

[16] G.W. Gibbons, M.B. Green, M.V. Perry, Instantons and seven-branes in type IIB superstring theory, Phys. Lett. B370, 37 (1996); hep-th/9511080.

[17] M. Bianchi, M.B. Green, S. Kovacs, G.C. Rossi, Instantons in supersymmetric Yang-Mills and D-instantons in IIB superstring theory, JHEP 9808, 13 (1998); hep-th/9807033.

[18] S.D. Mathur, A. Saxena, Y.K. Srivastava, Scalar propagator in the pp-wave geometry obtained from $\operatorname{Ad} S(5) \times S^{5}$, Nucl. Phys. B640, 367 (2002); hep-th/0205136.

[19] M. B. Green, M. Gutperle, Effects of D-instantons, Nucl. Phys. B498, 195 (1997); hep-th/9701093.

[20] M.B. Green, J.H. Schwarz, Supersymmetrical string theories, Phys. Lett. B109, 444 (1982).

[21] K. Skenderis, M. Taylor, Open strings in the plane wave background I: Quantization and symmetries, hep-th/0211011. 